\title{
Research on optimization of image fast feature point matching algorithm
}

\author{
Manyi $\mathrm{Wu}^{1,2}$
}

\begin{abstract}
The author studied the feature point extraction and matching based on BRISK and ORB algorithms, experimented with the advantages of both algorithms, and ascertained optimal pyramid layer and inter-layer scale parameters used in features extraction and matching for the same scale image and different scale images with BRISK and ORB algorithm, and analyzed the effectiveness of different parameters combinations on the accuracies of feature extraction and matching and proposed method to determine parameters based on the results. In addition, comparing with the traditional algorithm, using the optimal algorithm with the parameters combining Gaussian denoising, graying, and image sharpening, the ratio of feature points for detection improved 3\%; the number of effective matching points increased by nearly $2 \%$. Meanwhile, an algorithm experiment on UAV image mosaic was carried out. The transition of mosaic image color was more natural, and there was no clear mosaic joint with the stitching effect, which indicated that the optimized parameters and the extracted feature point pairs can be used for matrix operations and the algorithm is suitable for UAV image mosaic processing.
\end{abstract}

Keywords: BRISK and ORB algorithm, Fast feature detection, Algorithm optimization, UAV image mosaic processing

\section{Introduction}

Image feature point extraction and matching is a very important technical link in image processing. Image matching, image stitching, 3D (three dimension) modeling and other technical implementations rely on image feature point extraction and matching. After years of in-depth research and application practice, the algorithm for feature point extraction and description is constantly improving and perfecting. It has a wide range of applications in image matching $[1,2]$, image retrieval $[3,4]$, image recognition [5-7], video data tracking [8], image stitching [9], image classification [10], and many other aspects.

The image feature point extraction and matching algorithm is roughly divided into two types: descriptor-based matching algorithm and feature learning-based matching algorithm. This paper mainly studies the descriptor-based matching algorithm. The SIFT (scale-invariant feature

\footnotetext{
Correspondence: wuyurou2012@sina.com

${ }^{1}$ School of Geodesy and Geomatics, Wuhan University, Luoyu Road No.129, Wuhan, China

${ }^{2}$ The First Topographic Surveying Brigade of NASG (National Administration of Surveying, Mapping and Geoinformation of China), Cehui Road No.4, Xi'an, China
}

transform) [11] algorithm proposed by Lowe et al. has become the benchmark of this kind of algorithm because of its superior performance and stable performance, but the poor timeliness is the main obstacle affecting the application of the algorithm. In view of the poor timeliness of the SIFT algorithm, Bay et al. proposed the SURF (speeded up robust features) [12] algorithm, which is three to seven times $[12,13]$ more time efficient than the SIFT algorithm. The calculation timeliness of the ORB (object request broker) algorithm proposed by Rublee et al. [14] in 2011 is 100 times that of SIFT and 10 times that of SURF. The BRISK (binary robust invariant scalable keypoints) algorithm proposed by Leutenegger et al. [15] in 2011 is a feature extraction and binary description operator. It also has good rotation invariance, scale invariance, and robustness. Compared with SIFT, SURF, and other algorithms, the computational load is significantly reduced.

Of course, in order to pursue the timeliness of the algorithm, the accuracy of the algorithm is inevitably lost. Among them, BRISK and ORB are typical fast feature point detection and description algorithms, which have strong timeliness. The advantages of the two algorithms are strong robustness, good affine performance, and high 
timeliness. The disadvantages are that they do not have scale invariance and high error matching rate, which leads to some limitations of the algorithm.

Aiming at the problem that the feature point matching algorithm causes a high error matching rate in the scale invariance and the use of binary strings as feature descriptions, this paper aims to use BRISK and ORB algorithms as examples to study the improved algorithm and feature point extraction experiments based on BRISK, ORB algorithm feature point matching and the combination of the two advantages. The effects of different algorithm parameters and scale image sources on the accuracy of feature matching are obtained, and the optimal algorithm model parameters are proposed. Combined with Gaussian denoising, graying, and image sharpening enhancement, the algorithm is optimized to make the number of feature points and matching accuracy of the algorithm better.

\section{Related algorithms}

The BRISK algorithm and the ORB (Oriented FAST (features from accelerated segment test) and Rotated BRIEF (binary robust independent elementary features)) algorithm are fast feature extraction algorithms, and the computational load is significantly reduced compared with algorithms such as SIFT and SURF.

\subsection{FAST feature point extraction algorithm}

\subsubsection{Principle of FAST algorithm}

The FAST algorithm is a feature detection algorithm proposed by Rosten and Drummond [16], which is an improvement of the SUSAN (small univalue segment assimilating nucleus) corner extraction algorithm. It retains the SUSAN algorithm to detect the characteristics of various feature points $[17,18]$, and the algorithm has the advantages of fast detection speed and high accuracy of feature point detection. The algorithm is as follows:
As shown in Fig. 1, $p$ is the center pixel, taking the neighborhood radius as 3 pixels, and the pixels on the circumference are numbered clockwise 1 to 16 (denoted as $\mathrm{p} 1, \mathrm{p} 2, \ldots, \mathrm{p} 16)$. If the brightness of $\mathrm{N}$ consecutive pixels on the circumference is brighter than the center pixel brightness plus the threshold $t$, or darker than the center pixel brightness minus the threshold $t$, the center $\mathrm{p}$ pixel is called a corner point.

\subsubsection{Progress in FAST algorithm research}

In order to improve the detection speed, scholars have proposed to detect four points first. First compare the first and ninth pixels of Fig. 1 with the central pixel p. When the intensity is within the range of the threshold value $t$, it means that it is not a corner point, and then the fifth and third points are detected. If there are at least three points that are not similar to the center point, then the point can be judged as a corner point $[19,20]$. Finally, in order to improve the accuracy of the feature detection, all the 16 points of the determined corner point $\mathrm{P}$ are detected to accurately determine whether it is a corner point. Of course, this algorithm has the following shortcomings: (1) If only 2 of the four detection points are not similar to the center point, it is not clear that this is not a corner point; (2) the order of the detection points and the distribution around the corners will seriously affect the detection efficiency, and it is difficult to clearly select the pixel position to be the best reaction corner performance; (3) the results of the previous four-point test failed to fully use the latter test; and (4) feature points that are connected together are likely to detect adjacent locations.

In order to solve these problems, scholars have proposed several classic improvements. For example, the first to third questions can be solved by machine learning and the fourth problem can be solved by non-maximum suppression.

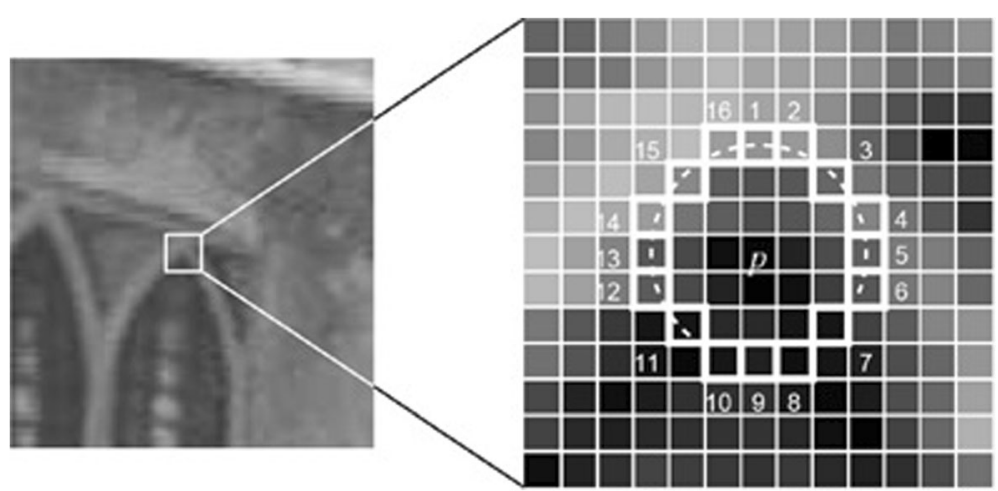

Fig. 1 FAST feature detection pixel number and detection range 

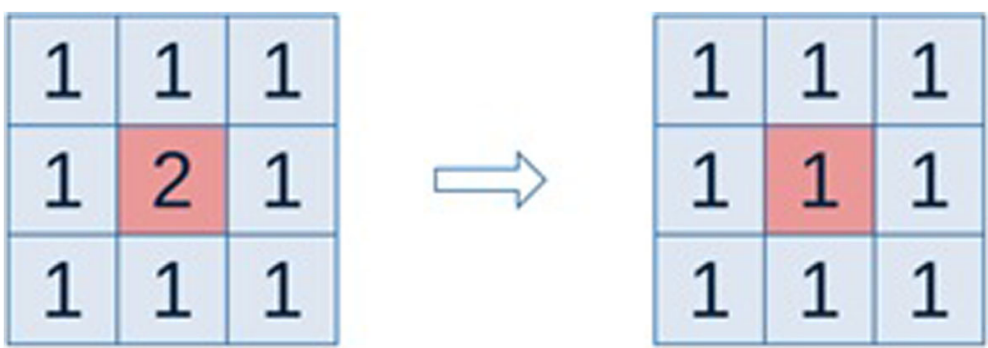

Fig. 2 Implementation process of Gaussian filtering algorithm

\subsection{BRIEF description}

\subsubsection{BRIEF description sub-principle}

The BRIEF description [21] operator describes the characteristics of these feature points in a certain way after detecting the corner points, which is called the descriptor of the feature points. Take the key point $\mathrm{P}$ as the center and $d$ as the radius, and arbitrarily select $N$ pairs in the circle. Take $N=4$ point pairs as an example to define an operation function as $T$, as shown in Eq. 2-1; then:

$$
\mathrm{T}(\mathrm{P}(\mathrm{A}, \mathrm{B}))=\left\{\begin{array}{c}
1 I_{A}>I_{B} \\
0 I_{A} \leq I_{B}
\end{array}\right.
$$

In the formula, $I_{A}$ and $I_{B}$ represent the gray value of the point.

In this way, the pair of points can be subjected to a $\mathrm{T}$ operation, and the obtained operation result of each point pair is composed into a final description operator, for example, any combination of 1101, 0011, etc., forms a binary description operator.

\subsubsection{Brief description of the research progress}

The ideal feature descriptor should be in the image of different size, direction, brightness, and darkness, and has a descriptor that is similar enough to be extracted, which is called reproducibility of the descriptor. That is to say, the descriptor is not sensitive to illumination (brightness), scale consistency (size), rotation consistency (angle), etc.; of course, this is only an ideal descriptor. The descriptors obtained by the BRIEF algorithm do not have these properties. Therefore, we must find ways to improve the algorithm in practical applications.

In terms of rotation consistency, we generally use the centroid method to obtain the image and regard the gray value of the image as a board with uneven density. The gray value is the quality of the pixel, and then, the center of mass $\mathrm{Q}$ is obtained. The feature point $\mathrm{P}$ is taken as the center of the circle, and PQ is used as the coordinate axis. At different rotation angles, the points taken out by the same point-taking mode are identical, which overcomes the problem of rotation consistency.

\subsection{Gaussian filtering algorithm}

Mean filtering, median filtering, and Gaussian filtering [22] are commonly used filtering algorithms for image noise processing. Mean filtering can only completely attenuate noise and cannot completely eliminate noise [23]; the median filter is sensitive to salt and pepper
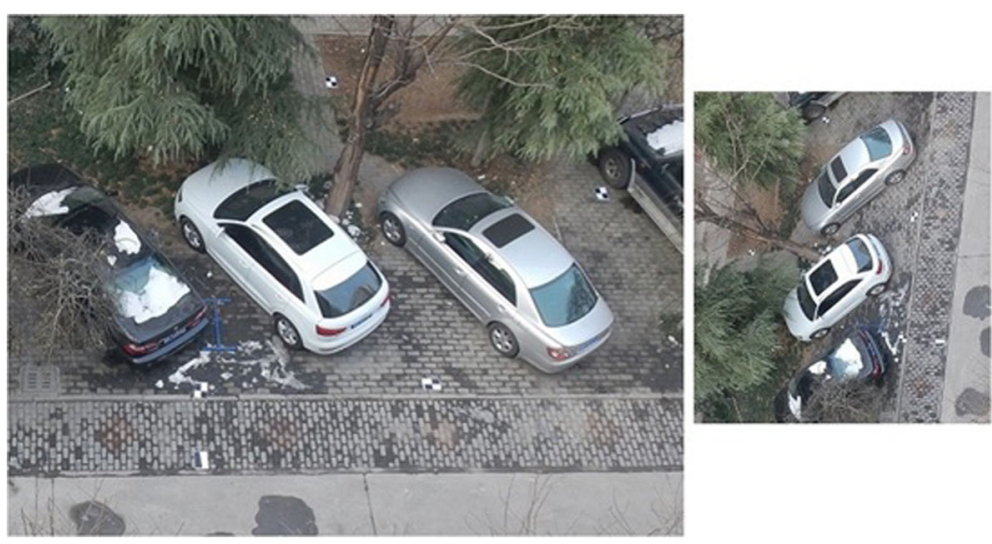

Fig. 3 Original image 

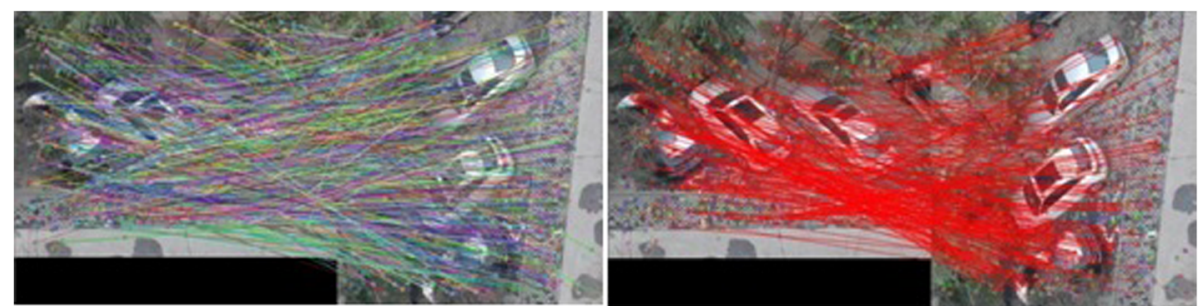

Fig. 4 BRISK algorithm for the same-scale image feature detection

noise and easily leads to image discontinuity [24, 25]. The Gaussian filtering algorithm smoothes the image while retaining the overall grayscale distribution of the image. The algorithm essentially convolves the (grayscale) image I with a Gaussian kernel, as shown in Eq. 2-2 below:

$$
I_{\sigma}=I * G_{\sigma}
$$

In the above formula, "*" represents a convolution operation, and $G_{\sigma}$ is a two-dimensional Gaussian kernel with a standard deviation of $\sigma$, as defined by Eq. 2-3:

$$
\mathrm{G}_{\sigma}=\frac{1}{2 \pi \sigma} \mathrm{e}^{-\left(\mathrm{x}^{2}+\mathrm{y}^{2}\right) / 2 \sigma^{2}}
$$

Convolution can be approximated as a weighted average process of pixel values in the image filtering process, as shown in Fig. 2.

\subsection{RANSAC algorithm}

RANSAC (random sample consensus) algorithm [26] is mainly used to filter out mismatching pairs in feature point detection. The idea of the algorithm is to use RANSAC algorithm to find a best homography matrix $\mathrm{H}$ (Homography Matrix) [27] with a matrix size of $3 \times 3$. The method of continuous iteration is used to find the optimal parameter model, and the points that do not conform to the optimal model are filtered out, so as to filter out the mismatched points [28]. The algorithm is implemented as follows:

Firstly, the transformation matrix is calculated using four sample data of random sampling and is recorded as model M;

Secondly, calculate the projection error of all the data in the data set and the model M. If the error threshold is less than the preset threshold, add the inner point set I; otherwise, filter out.

Again, if the current number of inner point set I elements is greater than the optimal inner point set I_best, then update I_best=I and update the iteration number $k$.

Finally, if the number of iterations is greater than $k$, then exit; otherwise, the number of iterations is incremented by 1 , and the above steps are repeated.

$$
k=\frac{\log (1-p)}{\log \left(1-w^{m}\right)}
$$

Table 1 Statistical table of the results of the gradual change of the scale parameters between the images when the pyramid layer is 4

\begin{tabular}{lllll}
\hline Pyramid image inter-scale parameter $(n)$ & Time $(\mathrm{ms})$ & Detection points & Match points & Accuracy $(\%)$ \\
\hline 1.0 & 328 & 541 & 200 & 37.0 \\
1.1 & 343 & 529 & 218 & 41.2 \\
1.2 & 327 & 515 & 241 & 46.8 \\
1.3 & 328 & 513 & 255 & 49.7 \\
1.4 & 328 & 512 & 257 & 50.2 \\
1.5 & 327 & 512 & 277 & 54.1 \\
1.6 & 343 & 505 & 280 & 55.4 \\
1.7 & 312 & 520 & 271 & 52.1 \\
1.8 & 312 & 504 & 272 & 54.0 \\
1.9 & 312 & 497 & 272 & 54.7 \\
2.0 & 296 & 475 & 259 & 54.5 \\
\hline
\end{tabular}




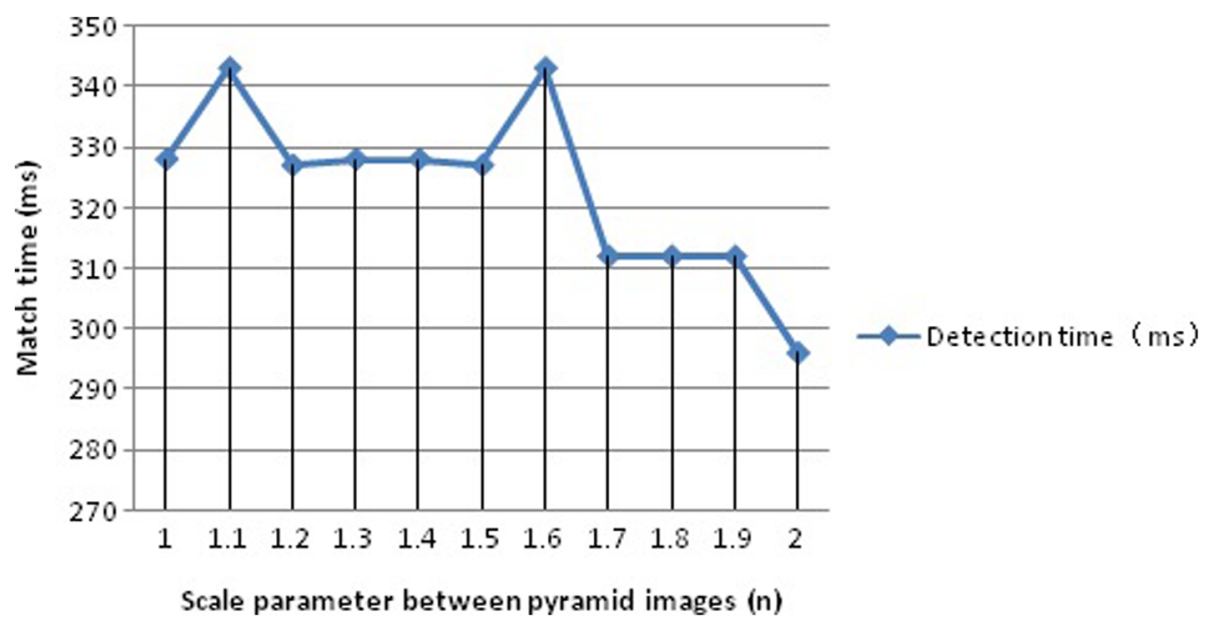

Fig. 5 Relationship between scale parameter variation and matching time between pyramid images

In the formula, $p$ is the confidence, $w$ is the ratio of "inner point", $m$ is the number of samples, the confidence is usually taken as 0.995 , and the sample is taken as the least.

\section{Method}

\subsection{Algorithm optimization}

On the one hand, the algorithm optimizes the optimal parameter combination of the optimal feature point extraction and matching of BRISK and ORB algorithms and proposes the optimal parameter combination according to different data source scale relationships. On the other hand, combined with BRISK, the ORB is optimized and improved, so that the feature point matching algorithm has the scale invariance of integrated BRISK and the robustness and fast computing power of the ORB algorithm.

\subsection{Algorithm optimization step}

In order to ensure the algorithm has better optimization effect, Gaussian algorithm is firstly used to filter out noise and image enhancement, then generate multi-scale space of image, and detect stable extreme points in multi-scale space, so that the extracted feature points have scale. Constant information. The feature points are then binary described using the BRIEF descriptor to generate binary descriptors with rotation invariance, scale invariance, and robustness. Finally, the RANSAC algorithm is used to filter out mismatched pairs and complete feature point matching.

The algorithm implementation steps are as follows:

Step 1: Read in the image to be detected and perform feature point detection

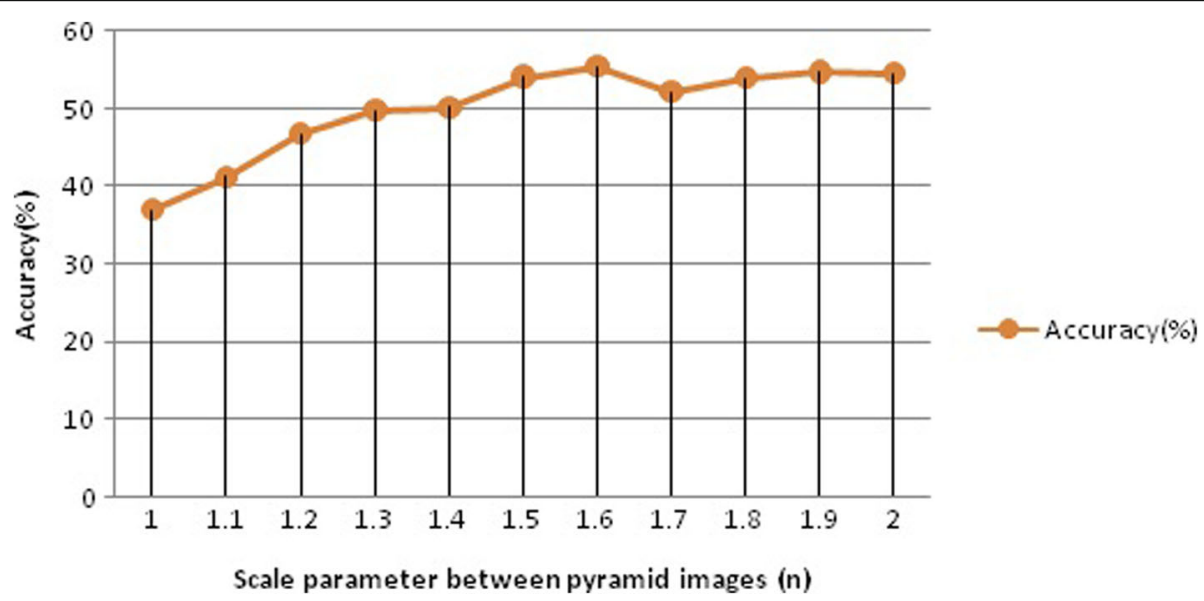

Fig. 6 Relationship between scale parameter variation and matching accuracy between pyramid images 


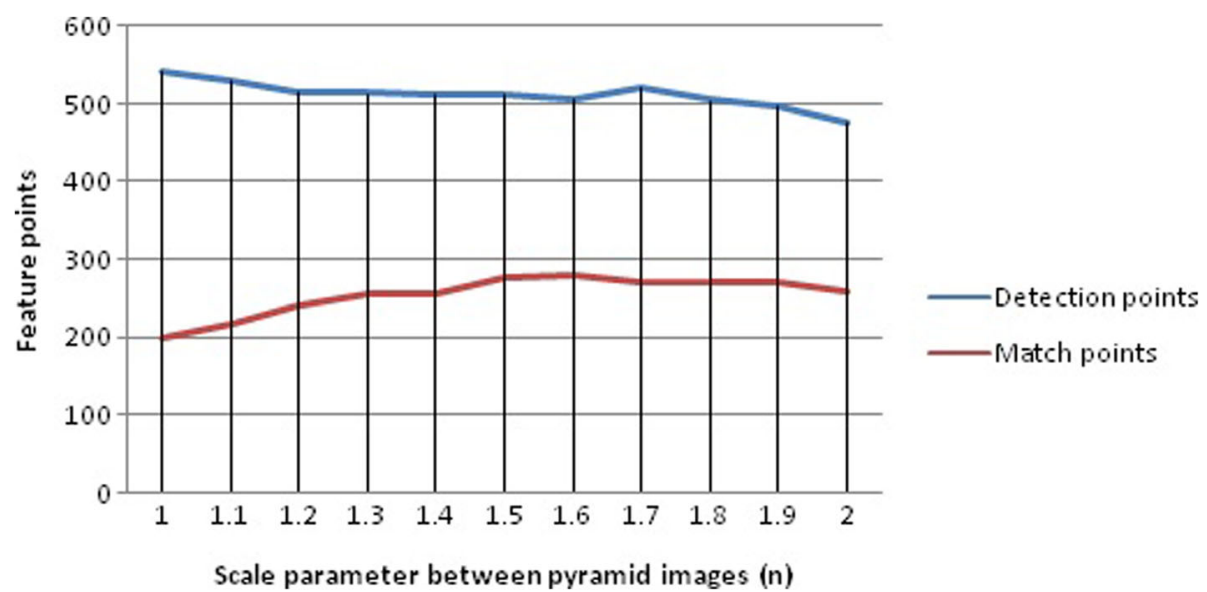

Fig. 7 Comparison of the number of feature points detected and the number of exact matches when the scale parameters of the pyramid image change

Construct a difference window, select the threshold $t$ and satisfy the Formula 2-5 to determine the feature corner:

$$
N=\sum_{\mathrm{x} \forall(\operatorname{circle}(p))}|I(x)-I(p)|>t
$$

In the formula, $I(p)$ is the gray value of the center of the circle, $I(x)$ is the gray value of any point on the circumference, and $t$ is the feature detection threshold. It is generally considered that three-quarters of the surrounding circle points are larger than a given threshold $t$, and it is judged that $p$ is a feature point, and all points in the image satisfying the above condition are recorded as the feature point set Q. In order to solve the problems of uneven distribution of characteristic corner points or dense corner points, non-maximum value suppression is used to enhance its robustness. The specific method is to first calculate the $V$ value of the feature point and then determine the $s$ value of multiple feature points in the neighborhood centered on the feature point $p$. If $p$ is the largest response value among all feature points in the neighborhood, it is reserved; otherwise, it is suppressed. The formula for calculating the $V$ value is as shown in Eq. 2-6:

$$
V=\max \left(\mid \sum(\text { pixel values }-p) \mid \text { if } \mid(\text { value }-p) \mid>t\right)
$$

In the formula, $V$ represents the score and $t$ represen the threshold.

Step 2: Create an image pyramid

First, re-sample the original image to generate a lower resolution image file and saving it. In order to form an image pyramid, lower resolution image sampling is sequentially performed until a predetermined layering is completed. Finally, the image of each layer is cut and saved into a slice file.

Table 2 Statistical results of experimental results with constant number of layers when the scale parameter between pyramid images is 1.6

\begin{tabular}{lllll}
\hline Pyramid layer $(i)$ & Time $(\mathrm{ms})$ & Detection points & Match points & Accuracy $(\%)$ \\
\hline 1 & 218 & 498 & 276 & 55.4 \\
2 & 281 & 506 & 283 & 55.9 \\
3 & 312 & 505 & 280 & 55.4 \\
4 & 405 & 504 & 280 & 55.6 \\
5 & 344 & 504 & 280 & 55.6 \\
6 & 328 & 504 & 280 & 55.6 \\
7 & 328 & 504 & 280 & 55.6 \\
9 & 327 & 504 & 280 & 55.6 \\
\hline
\end{tabular}




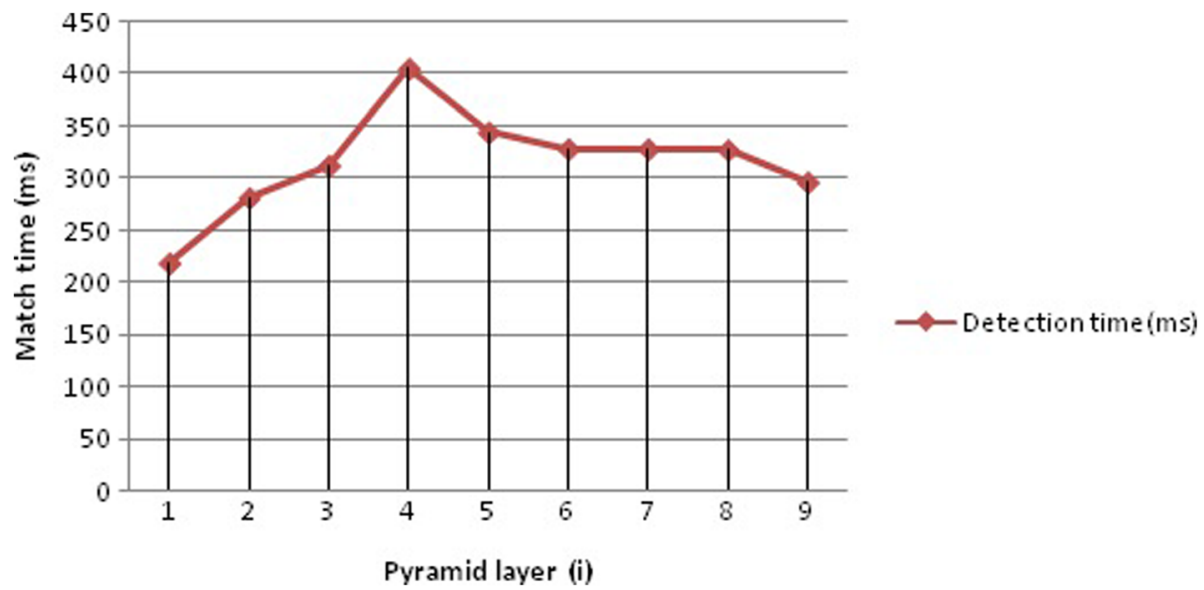

Fig. 8 Correspondence diagram between pyramid layer number and feature detection time

Next, in the scale space of each sample, the FAST algorithm is used, and the threshold value is taken to perform the FAST9-16 detection operator positioning and detection. In addition, in order to facilitate subsequent non-maximum suppression processing, a FAST5-8 corner detection is performed on the original image as a layer, which can be used to compare image feature points of adjacent scale spaces.

Step 3: Calculate the centroid direction of the feature points

$\mathrm{n}$ order to satisfy the rotation invariance, it is necessary to select the appropriate feature point main direction. $Q_{X}=\frac{M_{10}}{M_{00}}, Q_{Y}=\frac{M_{01}}{M_{00}}(Q$ is the pixel value centroid position) are calculated separately, and the feature point $P$ is fixed as a center of the circle and rotates as the object rotates. When we use PQ as the coordinate axis, the centroid direction $\theta$ of the feature point can be obtained by the calculation formula. Therefore, it is possible to take a consistent point in the same point mode under different rotation angles, which solves the problem of rotation consistency.

Step 4: Calculate the feature point descriptor

The exhaustive algorithm is used to calculate $n$ random point pairs, and the random point pairs are rotated according to the centroid direction of the feature points in the "third step." On the image pyramid layer corresponding to the feature points, the following formula is used to generate the binary descriptors:

$$
\tau(p: x, y)=\left\{\begin{array}{c}
1 p(x)<p(\mathrm{y}) \\
0 \text { otherwise }
\end{array}\right.
$$

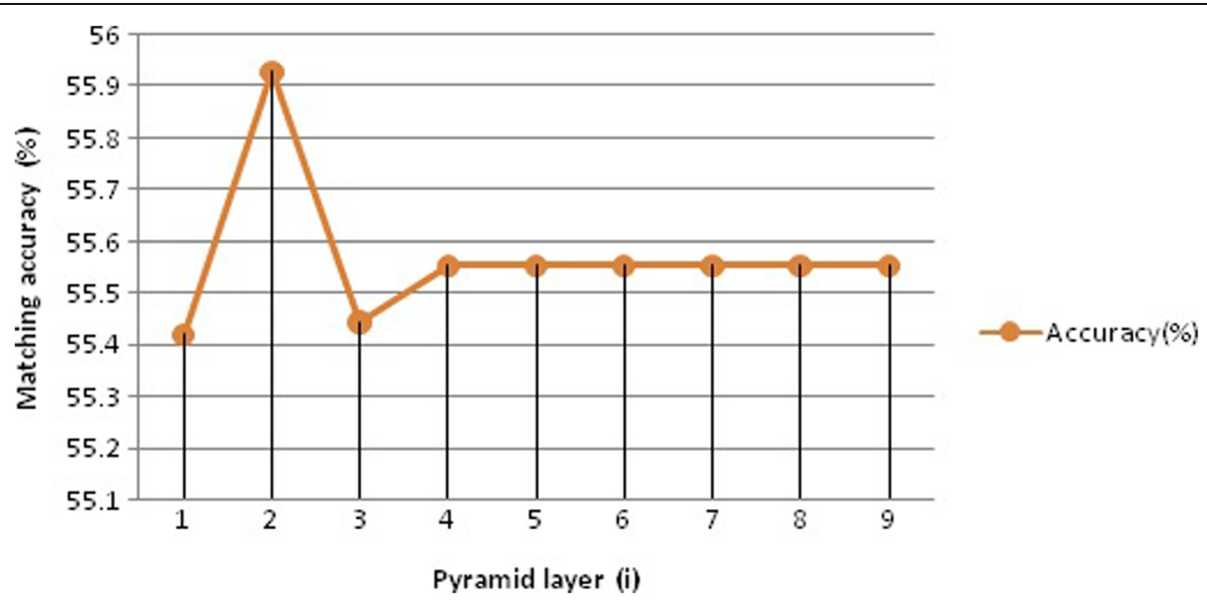

Fig. 9 Diagram of the number of pyramid layers and matching accuracy 


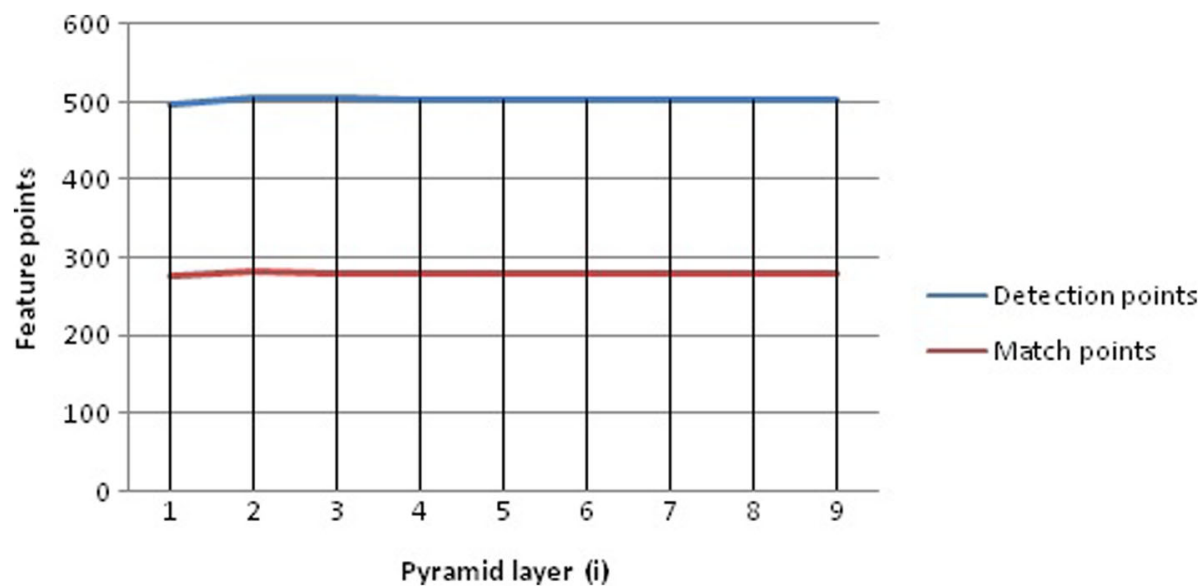

Fig. 10 Feature point detection number and exact matching number comparison chart when the scale parameter between pyramid images changes

In the formula, $(x, y)$ is a random point pair after rotation and $p(x)$ and $p(y)$ are the pixel values of random points $x=\left(u_{1}, v_{1}\right)$ and $y=\left(u_{2}, v_{2}\right)$ respectively.

Step 5: Feature point matching using $K$-nearest neighbor algorithm

Generally, $K$ is 2 or 3 . Calculate the Hamming distance from each feature point to all feature points to be matched. The two closest feature points to be matched are reserved, and the feature points form a matching point pair.

Step 6: Filter the feature point matching pairs and output the detected image

Matching the matching points in the previous step to the RANSAC algorithm with a threshold of 5 to filter out the mismatched points, find the optimal parameter matrix so that the number of data points satisfying the matrix is the largest, and use the iterative method to find the optimal parameter model. The point that does not conform to the optimal model is defined as "outer point" for filtering, so as to filter out the mismatched point.

\section{Algorithm optimization experiment results}

The computer configuration used in this experiment is Inter $(\mathrm{R})$ Core (TM) i7-3632QM, and the memory is 8.00 GB, 64-bit operating system. The feature matching algorithm experiment was performed using openCV in conjunction with the Visual Studio 2010 development program. The dataset selects images of the same scale in the experimental region and images that are transformed to generate different scales or rotate different angles, and perform feature matching experiments. The original image and the reduced and rotated image are shown in Fig. 3.

\subsection{Same scale feature point extraction experiment} Comparative analysis of BRISK and ORB algorithm in the same scale rotating $90^{\circ}$ image feature point extraction and different scale scaling $50 \%$ and rotating $90^{\circ}$ image feature point extraction time, feature point extraction number, accuracy, etc.

\subsubsection{Experiment 1: BRISK algorithm}

BRISK algorithm in the case of scale parameters $n(i=$ $1.1,1.2,1.3, \ldots, 2.0)$ between the layers $i(i=1,2, \ldots, 9)$ of different pyramids and pyramid images. The feature matching result uses the RANSAC algorithm with a
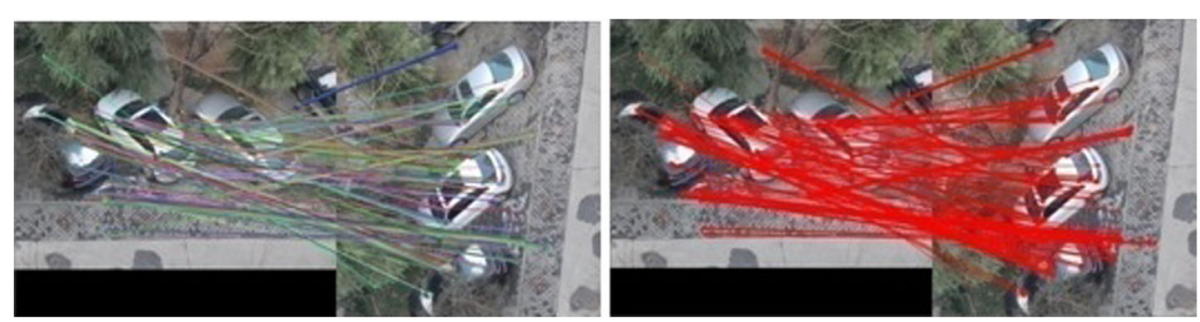

Fig. 11 Effect of the ORB algorithm on the same-scale image feature detection 
Table 3 Statistics table of the results of the scale parameter of the image when the pyramid layer is 8

\begin{tabular}{|c|c|c|c|c|}
\hline Pyramid image inter-scale parameter (n) & Time (ms) & Detection points & Match points & Accuracy (\%) \\
\hline 1.1 & 234 & 348 & 336 & 96.6 \\
\hline 1.2 & 203 & 368 & 350 & 95.1 \\
\hline 1.3 & 156 & 383 & 340 & 88.8 \\
\hline 1.4 & 141 & 347 & 297 & 85.6 \\
\hline 1.5 & 140 & 330 & 288 & 87.3 \\
\hline 1.6 & 125 & 321 & 284 & 88.5 \\
\hline 1.7 & 109 & 328 & 291 & 88.7 \\
\hline 1.8 & 109 & 314 & 272 & 86.6 \\
\hline 1.9 & 156 & 321 & 290 & 90.3 \\
\hline 2.0 & 94 & 320 & 278 & 86.9 \\
\hline
\end{tabular}

threshold of 5 to filter out the mismatched points and statistically characterize the experimental results. Experiments are carried out on the case where the image pyramid layer $i$ is constant, the inter-image scale parameter $n$ is gradually changed, the inter-image scale parameters are unchanged, and the pyramid layer number is gradually changed. The feature detection effect is shown in Fig. 4.

The experiment and results are as follows:

(1) The first case: using the conclusions in the paper "BRISK: Binary Robust Invariant Scalable

Keypoints", when the pyramid layer is 4, the feature points are detected when the scale parameters between the pyramid images are gradually changed from 1.1 to 2.0. Then, the RANSAC algorithm with a threshold of 5 filters out the mismatched points, and the results of the detection time, the number of feature points, and the number of feature points before and after filtering the mismatched points are shown in Table 1.
The relationship between the scale parameters between the pyramid images from 1.1, 1.2, ..., 2.0 and the feature detection time is shown in Fig. 5.

The relationship between the scale parameters of the pyramid image from 1.1, 1.2, ..., 2.0 and the accuracy of feature detection is shown in Fig. 6.

In the process of changing the scale parameters between pyramid images from 1.1, 1.2, ..., 2.0, the corresponding relationship between feature point detection and exact matching points is shown in Fig. 7.

Experimental summary:

1. As can be seen from Fig. 5, when the number of pyramid layers of the same scale image is 4 , the scale parameter $n$ between the pyramid images changes from 1.1, 1.2, ..., 2.0. The time consumed by feature detection is generally decreasing, with a maximum of $343 \mathrm{~ms}$ and a minimum of $296 \mathrm{~ms}$.

2. It can be seen from Fig. 6 that when the number of pyramid layers of the same scale image is 4 , during

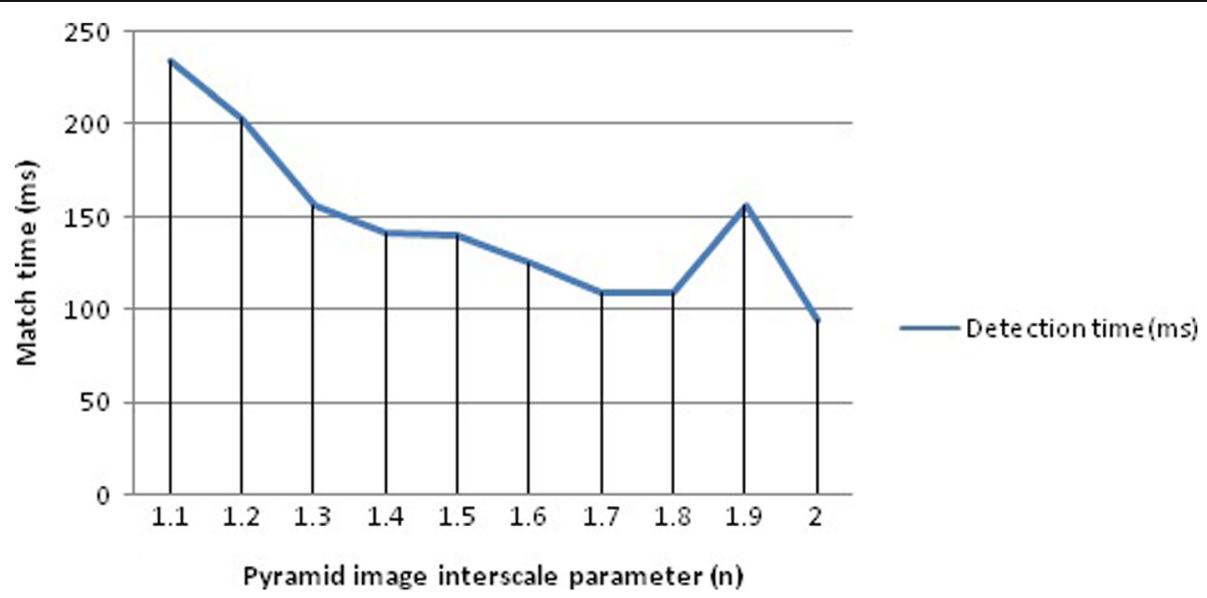

Fig. 12 Time-dependent relationship between scale parameter variation and feature detection in pyramid images 


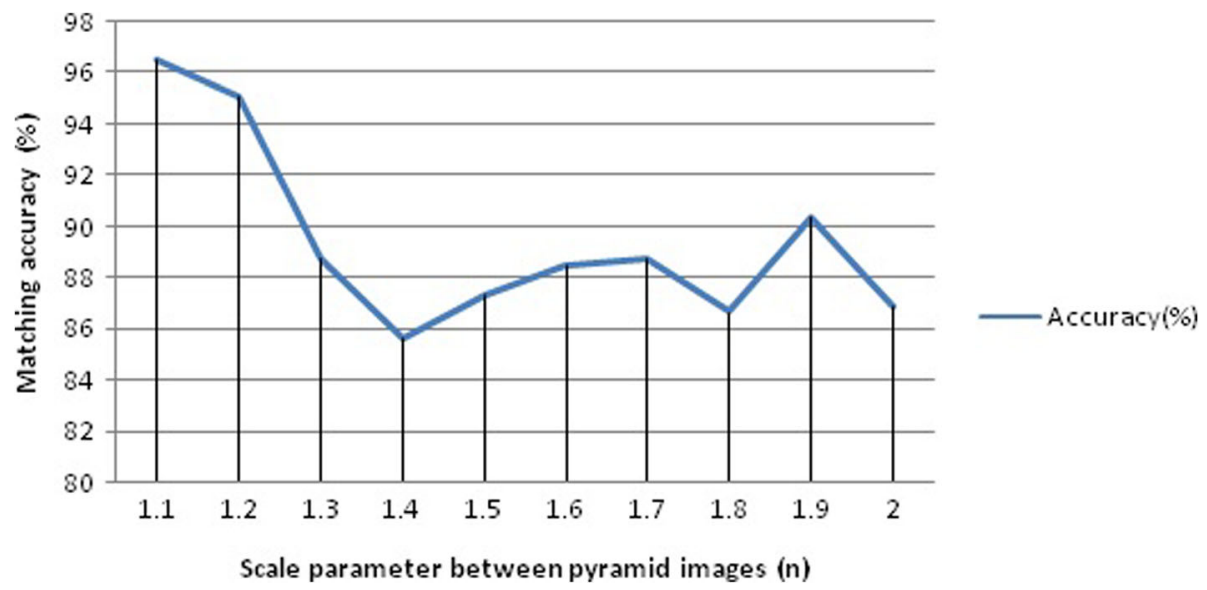

Fig. 13 Relationship between scale parameter variation and feature detection accuracy of pyramid images

the change of the scale parameter $n$ between the pyramid images from $1.1,1.2, \ldots, 2.0$, the accuracy of feature detection gradually increased, and it gradually stabilized from 37 to $54 \%$.

3. It can be seen from Fig. 7 that when the number of pyramid layers of the same scale image is 4 , during the change of the scale parameter $n$ between the pyramid images from 1.1, 1.2, ..., 2.0, the number of feature points detected is gradually decreasing, and the number of feature points that are accurately matched is gradually increasing.

(2) The second case: from the "first case" experiment results of "Experiment 1", it is known that in the case of the same scale image, the feature matching accuracy is higher when the number of pyramid layers is 4 and the scale parameter between pyramid images is 1.6. Therefore, when the pyramid scale parameter is set to 1.6 , the number of pyramid layers $\mathrm{i}(i=1,2, \ldots, 9)$ is gradually changed from 1 to 9 for experiment. Finally, the RANSAC algorithm with a threshold of 5 is used to filter out the mismatched points. The experimental results are shown in Table 2.

When the scale parameter between pyramid images is 1.6, the correspondence between the number of pyramid layers $i$ and the detection time is shown in Fig. 8.

When the scale parameter between pyramid images is 1.6 , the relationship between the number of pyramid layers $i$ and the detection accuracy is shown in Fig. 9.

When the scale parameter between pyramid images is 1.6, the correspondence between the number of feature point detection points and the number of valid matching points in the process of pyramid change from 1 to 9 is shown in Fig. 10.

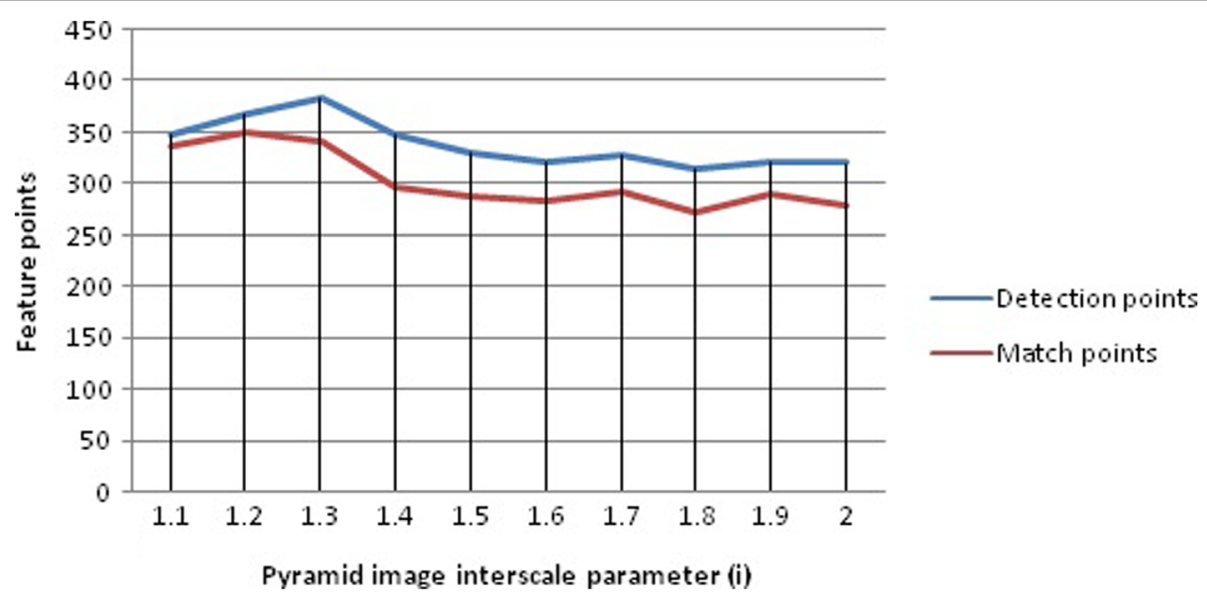

Fig. 14 Comparison of the number of feature points detected and the number of exact matches when the scale parameters of the pyramid image change 
Table 4 Statistical results of experimental results when the scale parameter of the pyramid image is 1.1

\begin{tabular}{lllll}
\hline Pyramid layer $(i)$ & Time $(\mathrm{ms})$ & Detection points & Match points & Accuracy $(\%)$ \\
\hline 1 & 109 & 341 & 290 & 35.0 \\
2 & 109 & 349 & 328 & 94.0 \\
3 & 203 & 348 & 332 & 95.4 \\
4 & 172 & 348 & 334 & 96.0 \\
5 & 265 & 348 & 334 & 96.0 \\
6 & 219 & 352 & 340 & 96.6 \\
7 & 281 & 348 & 337 & 96.8 \\
9 & 234 & 348 & 336 & 96.6 \\
\hline
\end{tabular}

Experimental summary:

1. It can be seen from Fig. 8 that when the inter-scale image parameter of the pyramid image of the same scale image is 1.6 , the consuming time of feature detection generally increases gradually during the gradual change of the number of pyramid layers from 1 to 9 . However, when the number of pyramid layers is 7 to 9 layers, the consumption time has a tendency to fall.

2. As can be seen from Fig. 9, when the scale parameter of the pyramid image of the same scale image is 1.6, the accuracy of the overall feature detection of the BRISK algorithm is stable at about 55\% during the change of the pyramid layer from 1 to 9 . The fluctuation of the layer number is 1 to 4 .

3. As can be seen from Fig. 10, when the scale parameter of the pyramid image of the same scale image is 1.6, during the change of the number of pyramid layers from 1 to 9 , the number of feature points detected and the number of feature points that match exactly do not change.
From the experimental statistics of the above two cases, the following conclusions can be drawn:

When the pyramid layer is 4 in the BRISK algorithm, the matching accuracy of the pyramid image between the same scale image is 1.6 , which is about $54 \%$. The change of the number of pyramid layers slightly increases the feature detection time and has no significant influence on the number of features detected and the matching accuracy. It shows that the BRISK algorithm pyramid layer change has little effect on the feature point detection number and matching accuracy.

\subsubsection{Experiment 2: ORB algorithm}

ORB algorithm performs feature matching in the case of different pyramid layer numbers $i(i=1,2, \ldots, 9)$ and pyramid image inter-scale parameter $n(n=1.1,1.2, \ldots$, 2.0). And use the RANSAC algorithm with a threshold of 5 to filter out the mismatch points. The experiment was carried out in the same way as in "Section 4.1.1", and the feature point detection results were counted.

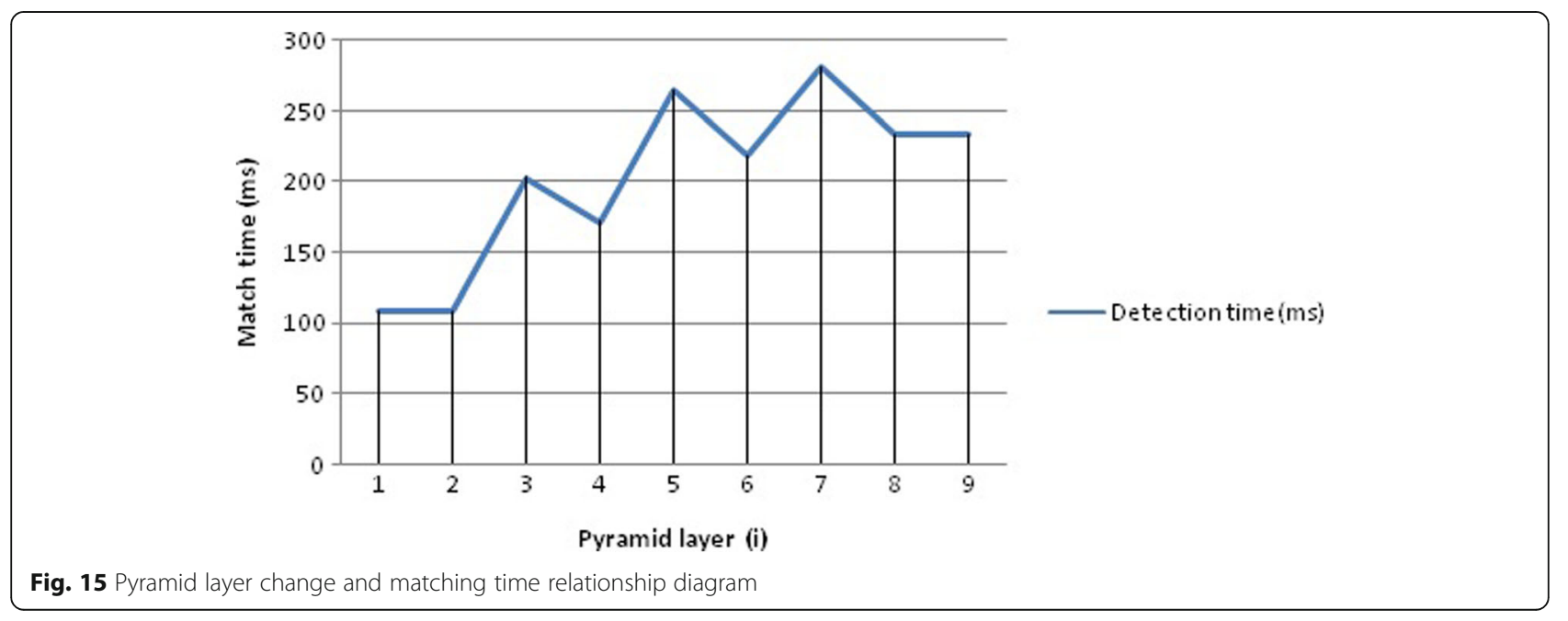




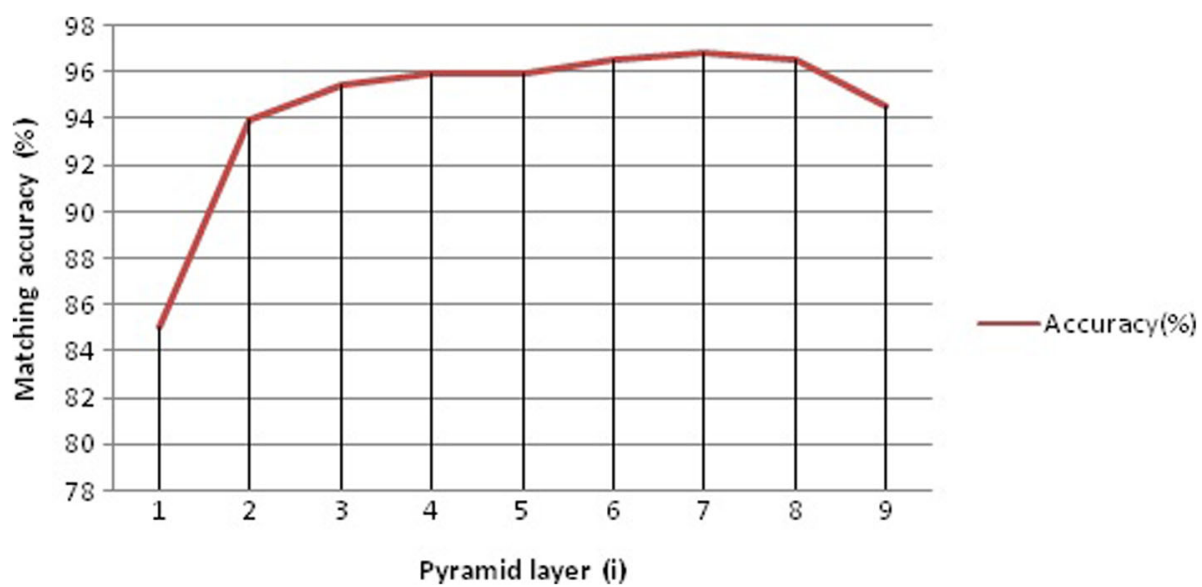

Fig. 16 Diagram of pyramid layer change and matching accuracy

(1) The first case: the conclusion of the paper in the paper "ORB: an efficient alternative to SIFT or SURF", the number of pyramid layers is 8 and the feature points are detected when the scale parameter of the pyramid image changes gradually from 1.1 to 2.0. The RANSAC algorithm with a threshold of 5 filters out the mismatch points. The experimental results are shown in Fig. 11.

Table 3 shows the results of detection time, number of feature points, and number of feature points before and after filtering the mismatched points in the ORB algorithm feature detection.

The relationship between the scale parameter of the pyramid image from 1.1, 1.2, ..., 2.0 and the time consumption of feature detection is shown in Fig. 12.

The relationship between the gradual change of the scale parameter of the pyramid image between 1.1, 1.2,
..., 2.0 and the accuracy of feature detection matching is shown in Fig. 13.

When the scale parameter between pyramid images changes between 1.1, 1.2, ..., 2.0, the correspondence between feature point detection and exact matching points is shown in Fig. 14.

Experimental summary:

1. It can be seen from Fig. 12 that when the number of pyramid layers of the same scale image is 8 , the scale parameter $n$ between the pyramid images changes from 1.1, 1.2, ..., 2.0. The ORB algorithm feature detection consumption time is gradually decreasing, which takes up to $234 \mathrm{~ms}$ and the minimum time is $94 \mathrm{~ms}$.

2. It can be seen from Fig. 13 that when the number of pyramid layers of the same scale image is 8 , when the scale parameter $n$ between the pyramid

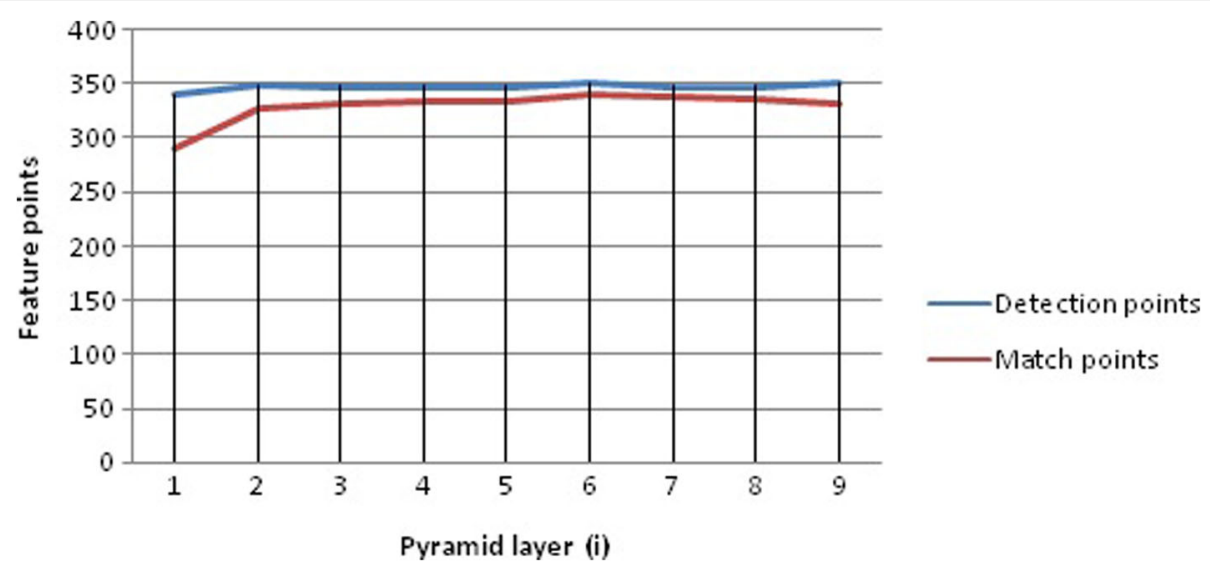

Fig. 17 Comparison of the number of feature points detected and the number of exact matches when the number of pyramid layers changes gradually 

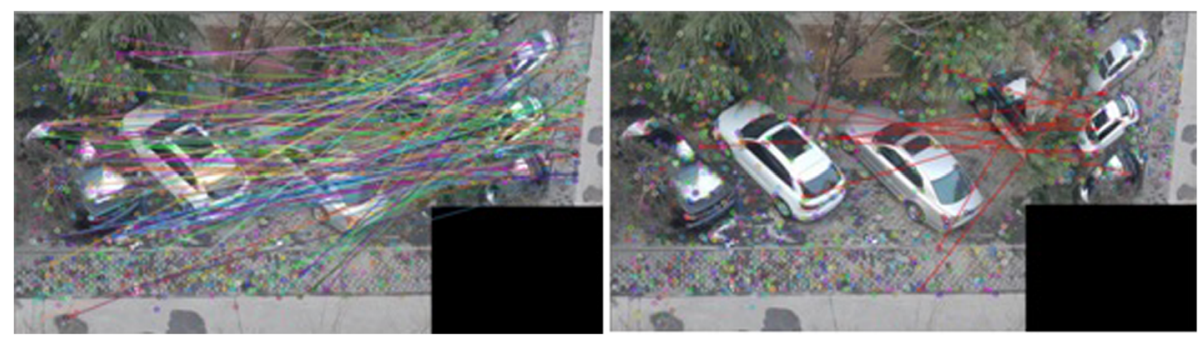

Fig. 18 Results of BRISK algorithm in image detection of different scales

images changes from 1.1, 1.2, ..., 2.0, the accuracy of feature detection of ORB algorithm is gradually decreasing, decreasing from 96 to $87 \%$. During the change of scale parameters between pyramid images from 1.4 to 2.0, the matching accuracy fluctuates greatly and fluctuates around $87 \%$.

3. It can be seen from Fig. 14 that when the number of pyramid layers of the same scale image is 8 , the scale parameter $n$ between the pyramid images changes from 1.1, 1.2, ..., 2.0. The number of feature points and the number of exact matches are gradually decreasing, and the trend is reduced.

(2) The second case: from the results of the "first case" experiment of "Experiment 2", when the number of pyramid layers in the same scale image is 8 and the scale parameter between the pyramid images is 1.1, the ORB algorithm has a high feature matching accuracy, but this method is time consuming. Because this paper mainly studies the optimization method to improve the accuracy of feature detection, the feature detection experiment with different scales of different gold characters is selected with the scale parameter of 1.1. Finally, the mismatching point is filtered by the

RANSAC algorithm with a threshold of 5 . The experimental results are statistically as follows (Table 4).

When the scale parameter between pyramid images is 1.1 , the relationship between the number of pyramid layers $i$ from 1 to 9 and the detection time is shown in Fig. 15.

When the scale parameter between pyramid images is 1.1, the relationship between the number of pyramid layers changing from 1 to 9 and the detection accuracy is shown in Fig. 16.

When the number of pyramid layers changes from 1 to 9 , the correspondence between the number of feature point detection points and the exact matching points is shown in Fig. 17.

Experimental summary:

1. As can be seen from Fig. 15, when the scale parameter between pyramid images of the same scale image is 1.1, during the change of the pyramid layer number i from 1 to 9 , the ORB algorithm feature detection consumption time gradually increases, with the longest time of $281 \mathrm{~ms}$ and the minimum time of $109 \mathrm{~ms}$.

2. As can be seen from Fig. 16, when the scale parameter between the pyramid images of the

Table 5 Statistics table of the results of the constant change of the inter-image scale parameters when the pyramid layer is 4

\begin{tabular}{|c|c|c|c|c|}
\hline Scale parameter between pyramid images $(n)$ & Time (ms) & Detection points & Match points & Accuracy (\%) \\
\hline 1.1 & 328 & 170 & 9 & 5.3 \\
\hline 1.2 & 328 & 161 & 8 & 5.0 \\
\hline 1.3 & 328 & 169 & 8 & 4.7 \\
\hline 1.4 & 312 & 165 & 14 & 8.5 \\
\hline 1.5 & 171 & 166 & 14 & 8.4 \\
\hline 1.6 & 172 & 144 & 22 & 15.3 \\
\hline 1.7 & 171 & 144 & 14 & 9.7 \\
\hline 1.8 & 156 & 148 & 7 & 4.7 \\
\hline 1.9 & 312 & 136 & 8 & 5.9 \\
\hline 2.0 & 343 & 135 & 8 & 5.9 \\
\hline
\end{tabular}




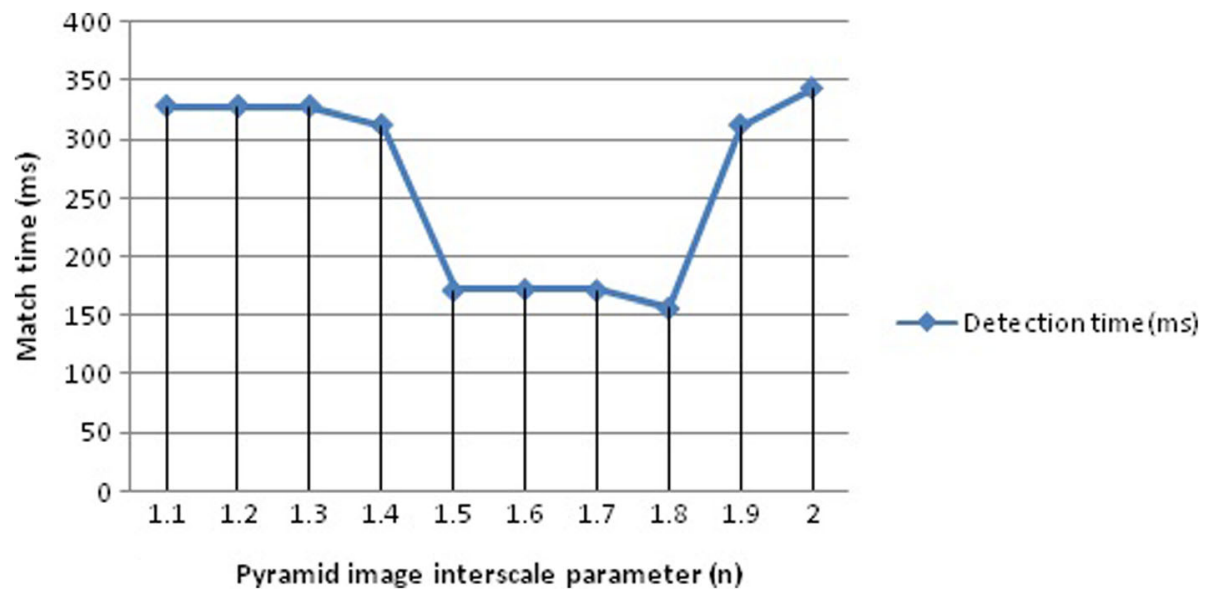

Fig. 19 Time-dependent relationship between scale parameter variation and matching between pyramid images

same scale image is 1.1, the accuracy of the feature detection of the ORB algorithm gradually increases during the change of the number of pyramid layers i from 1 to 9 , with a minimum of $85 \%$ and a maximum close to $97 \%$.

3. As can be seen from Fig. 17, when the scale parameter between the pyramid images of the same scale image is 1.1, the number of feature points and the number of exact matches are relatively stable during the change of the number of pyramid layers i from 1 to 9 , which is basically a horizontal trend.

From the statistics of the experimental results of the above two cases, the following conclusions can be drawn:

When the pyramid layer is 8 , when the scale parameter between the image pyramid images of the same scale is 1.1, the matching accuracy of the ORB algorithm is relatively high, about $97 \%$. However, this is the most time consuming, and the number of detection points and the number of exact matches are weakly related to the change in the number of pyramid layers.

\subsection{Feature point extraction experiment of different scale images \\ 4.2.1 Experiment 3: BRISK algorithm}

For the feature matching result of BRISK algorithm in the case of different pyramid layer number $i(i=1,2$, ..., 9) and different pyramid image size parameter $n$ $(n=1.1,1.2,1.3, \ldots, 2.0)$, the RANSAC algorithm with threshold value of 5 is used to filter out the mismatching point. The experiment is also carried out according to the "Section 4.1" chapter in two cases. The effect of feature detection and filtering mismatch is shown in Fig. 18.

The experimental development and results are as follows:

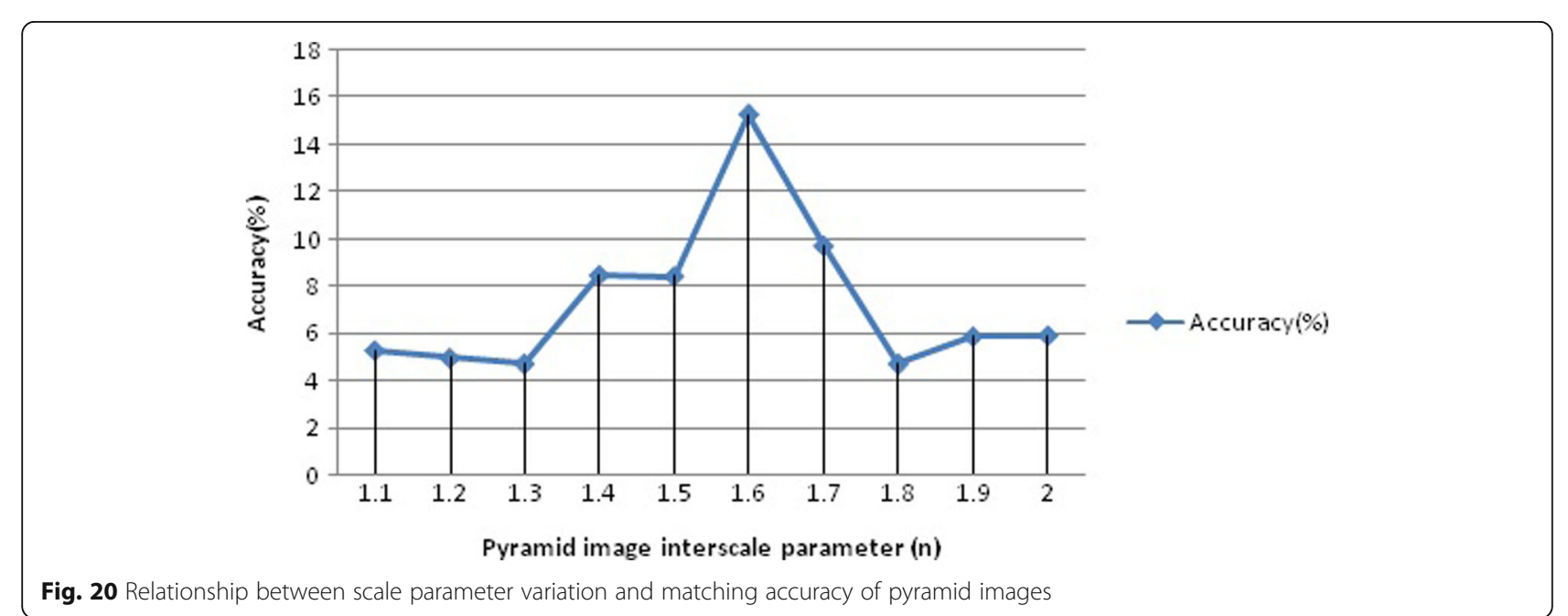




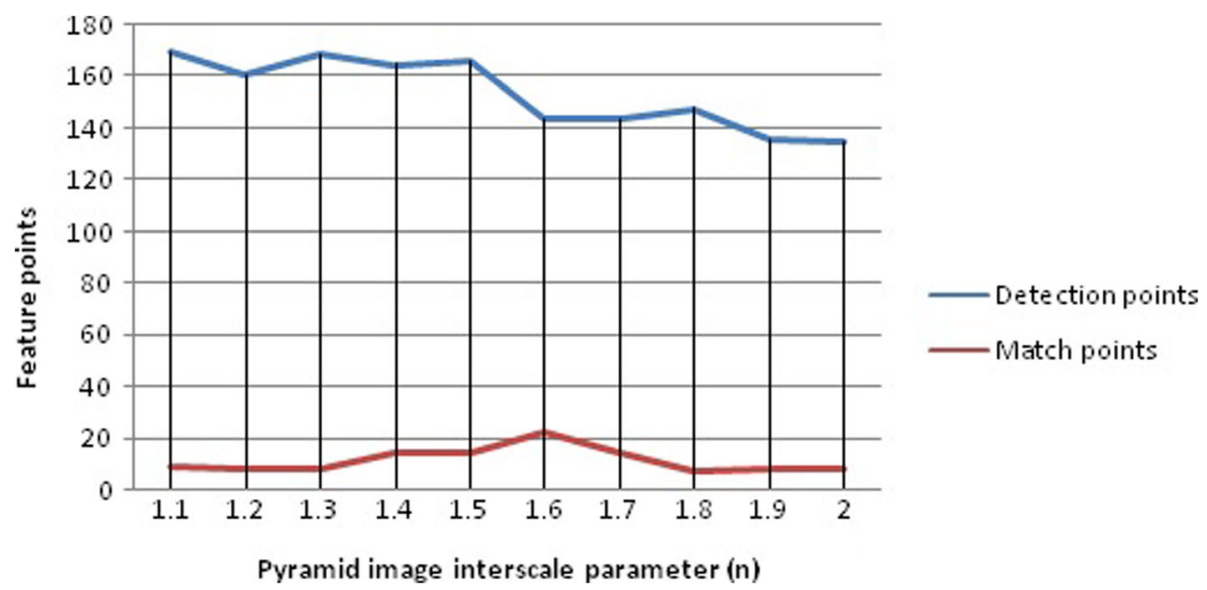

Fig. 21 Comparison of the number of feature points detected and the number of exact matches when the scale parameters of the pyramid image change

(1) The first case: consistent with the idea in the "Section 4.1.1", select the pyramid layer to be 4 and perform feature point detection under the scale parameter $n(n=1.1,1.2, \ldots, 2.0)$ between the pyramid images, and use the RANSAC algorithm with a threshold of 5 to filter out the mismatched points. Table 5 shows the results of detection time, number of feature points, and number of feature points before and after filtering of mismatched points during feature detection:

When the number of pyramid layers is 4 , the relationship between the scale parameters between the pyramid images from 1.0, 1.1, ..., 2.0 and the feature detection time is shown in Fig. 19.

When the number of pyramid layers is 4 , the relationship between the scale parameters between the pyramid images from 1.0, 1.1, ..., 2.0 and the accuracy of feature detection is shown in Fig. 20.
When the number of pyramid layers is 4 , the relationship between the number of feature point detection points and the exact matching points is shown in Fig. 21.

Experimental summary:

1. As can be seen from Fig. 19, images of different scales (50\% difference between the left and right image scales). The pyramid layer is 4 and the scale parameter of the pyramid image changes from 1.1 to 2.0. When the scale parameter is between 1.5 and 1.8, it takes less time, and the maximum time is $343 \mathrm{~ms}$ and the minimum time is $156 \mathrm{~ms}$.

2. As can be seen from Fig. 20, images with different scales (the difference between the left image and the right image scale is 50\%) and the pyramid layer is 4 , during the change of the scale parameter between the pyramid images from 1.1 to 2.0, except that the scale parameter between the pyramid images is 1.6, the detection accuracy is $16 \%$, and

Table 6 Statistical results of the experimental results of the ever-changing pyramid layer when the scale parameter between pyramid images is 1.6

\begin{tabular}{lllll}
\hline Pyramid layer $(i)$ & Time $(\mathrm{ms})$ & Detection points & Match points & Accuracy $(\%)$ \\
\hline 1 & 266 & 149 & 10 & 12 \\
2 & 202 & 146 & 22 & 8.2 \\
3 & 172 & 144 & 22 & 15.3 \\
4 & 172 & 144 & 22 & 15.3 \\
5 & 171 & 144 & 22 & 15.3 \\
6 & 312 & 144 & 22 & 15.3 \\
7 & 219 & 144 & 22 & 15.3 \\
8 & 234 & 144 & 22 & 15.3 \\
9
\end{tabular}




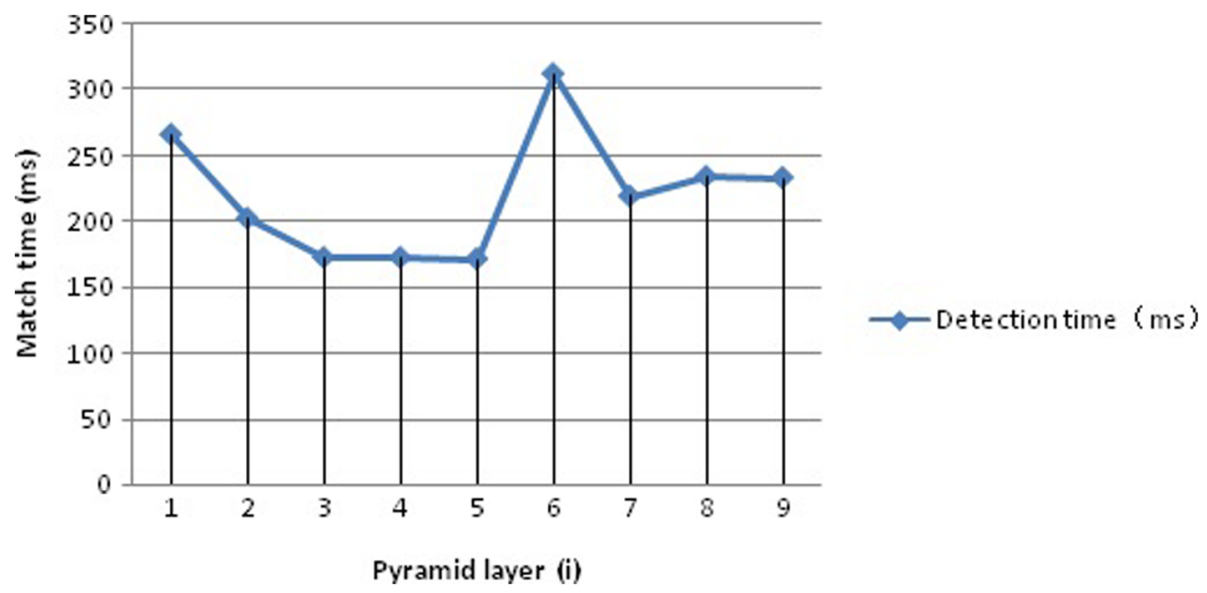

Fig. 22 Pyramid layer change and matching time relationship diagram

the detection accuracy of other parameters is less than $10 \%$.

3. As can be seen from Fig. 21, the images of different scales (the difference between the left image and the right image scale are 50\%) and the pyramid layer is 4 , during the change of the scale parameter between the pyramid images from 1.1 to 2.0, the number of feature points is very different from the number of exact matches. The algorithm has obvious defects and there is much room for improvement.

(2) The second case: from "Experiment 1 of Section 4.2.1", when the scale parameter between pyramid images is 1.6 , the matching accuracy is relatively high. Therefore, the pyramid scale parameter is 1.6 , and the number of layers $i(i=1,2, \ldots, 9)$ of the pyramid is gradually changed for experiment. Finally, the mismatch point is filtered by the
RANSAC algorithm with a threshold of 5 . The experimental results are statistically as follows (Table 6).

When the scale parameter between the pyramid images is 1.6, the relationship between the pyramid layer number $i$ and the feature detection time is shown in Fig. 22.

The scale parameter between the pyramid images is 1.6, and the relationship between the pyramid layer number $i$ and the detection accuracy when changing from 1 to 9 is shown in Fig. 23.

In the process of changing the scale parameter between pyramid images from 1 to 9 , the correspondence between the number of points detected by the feature points and the number of exact matching points is shown in Fig. 24.

Experimental summary:

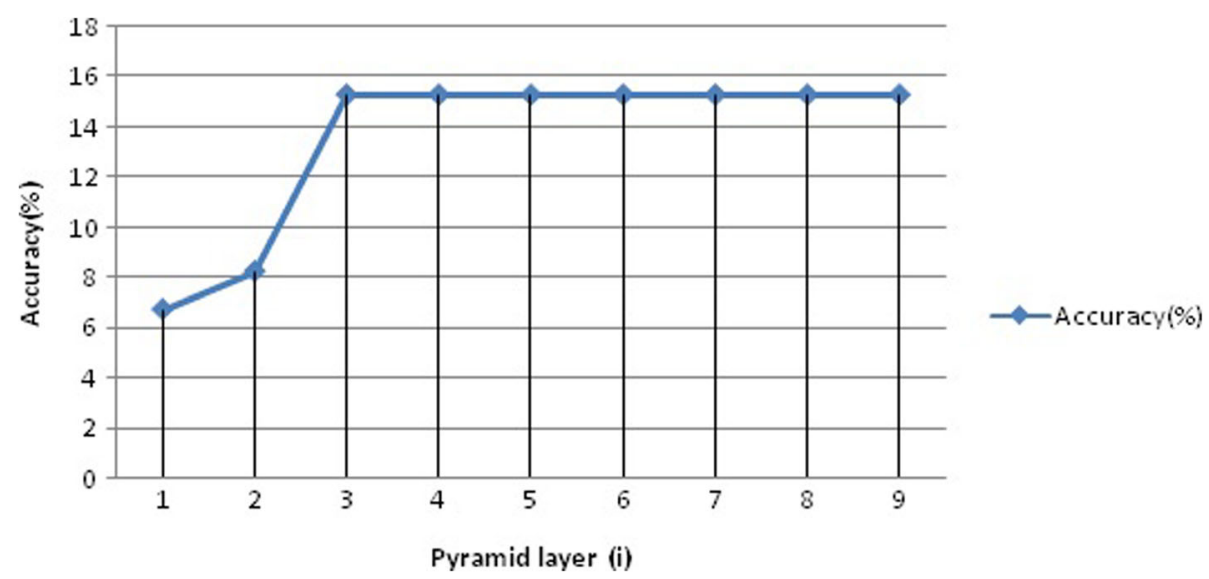

Fig. 23 Diagram of the change of the number of layers of the pyramid and the matching accuracy 


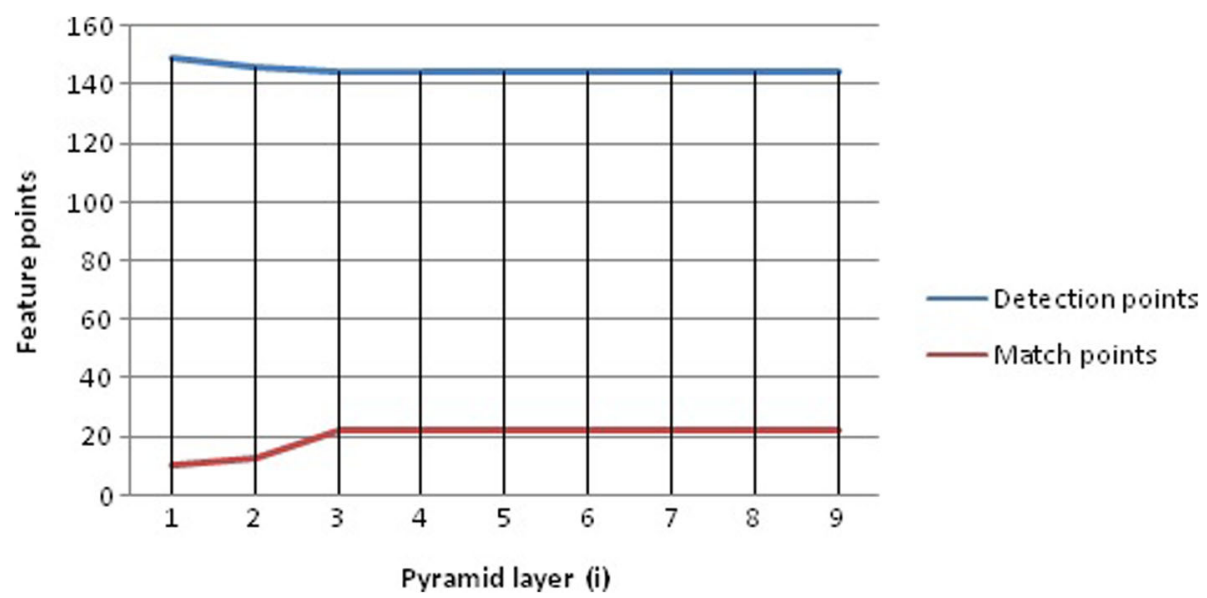

Fig. 24 Comparison of the number of feature points detected and the number of exact matches when the number of pyramid layers changes

1. As can be seen from Fig. 22, in the process of the pyramid layer changing from 1 to 9 , when the scale between the different scales (the left image and the right image scale is 50\%) and the scale parameter between the pyramid images is 1.6, the feature point detection takes time and fluctuates greatly. The maximum time is $312 \mathrm{~ms}$ and the minimum time is $172 \mathrm{~ms}$.

2. As can be seen from Fig. 23, when the scale parameter between the pyramid scales is 1.6 and the scale parameter between the pyramid images is 1.6 , the detection accuracy is stable at around 16\%. When the pyramid layer is 1 to 3 , the feature detection accuracy increases significantly with the increase of the number of layers.

3. As can be seen from Fig. 24, when the pyramid layer changes from 1 to 9 , the image with different scales (the difference between the left image and the right image scale is 50\%) and the scale parameter between the pyramid images is 1.6, the contrast between the number of feature points and the exact number of matches is very large. The algorithm has the drawback of insufficient detection accuracy.

From the statistics of the experimental results of the above two cases, the following conclusions can be drawn:

In the process of changing the scale parameter of the pyramid image from 1.1 to 2.0 , when the pyramid layer is 4 , the image of different scales (the difference between the left image and the right image scale is $50 \%$ ), the average matching accuracy of the BRISK algorithm is less than $10 \%$. The matching accuracy is only $16 \%$ when the scale parameter between the pyramid images is 1.6; when the pyramid layer changes from 1 to 9 , when the scale parameter between the pyramid images is 1.6, the average matching accuracy is lower than $16 \%$. In the process of increasing the pyramid layer from 1 to 3 , the matching accuracy increases to about $15 \%$, and between 4 and 9 layers, the matching accuracy tends to be stable.
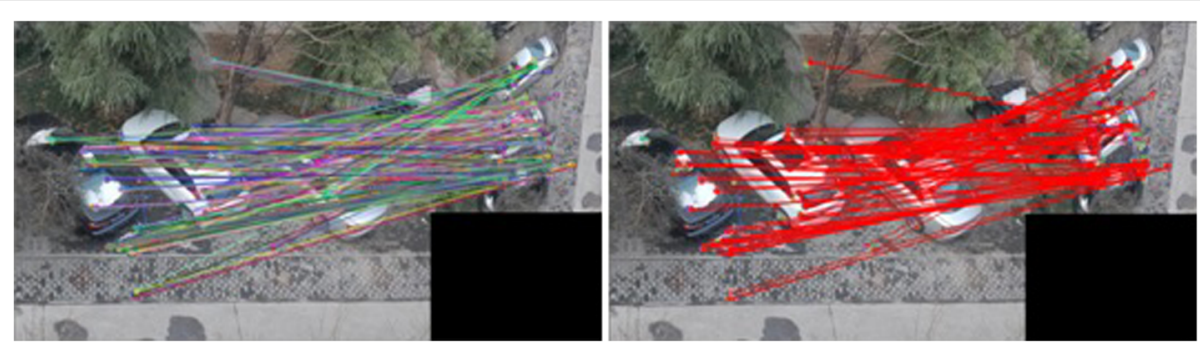

Fig. 25 Result graph of ORB algorithm in image detection of different scales 
Table 7 Statistics table of the results of the constant change of the inter-image scale parameters when the pyramid layer is 8

\begin{tabular}{lllll}
\hline Scale parameter between pyramid images $(n)$ & Time $(\mathrm{ms})$ & Detection points & Match points & Accuracy (\%) \\
\hline 1.1 & 219 & 230 & 62 & 149 \\
1.2 & 125 & 265 & 160 & 56.2 \\
1.3 & 124 & 264 & 154 & 60.6 \\
1.4 & 109 & 251 & 128 & 61.4 \\
1.5 & 125 & 238 & 93 & 53.8 \\
1.6 & 93 & 245 & 112 & 37.9 \\
1.7 & 78 & 234 & 120 & 47.9 \\
1.8 & 93 & 246 & 131 & 48.8 \\
1.9 & 78 & 247 & 137 & 53.0 \\
2.0 & 78 & 252 & 54.6 & \\
\hline
\end{tabular}

\subsubsection{Experiment 4: ORB algorithm}

Testing the feature matching results of the ORB algorithm in the case of the number of layers $i(i=1,2, \ldots, 9)$ of different pyramids and the scale parameter $n(n=1.1$, $1.2, \ldots, 2.0)$ between different pyramid images, the RANSAC algorithm with a threshold of 5 is selected to filter out the mismatched points, and the experimental results of the feature points are statistically analyzed. The experimental results are shown in Fig. 25.

The experimental development and results are as follows:

(1) The first case: when the pyramid layer is 8 , the scale parameter of the pyramid image gradually changes from 1.1 to 2.0 for feature point detection. The results of the detection time, the number of feature points, and the number of feature points before and after filtering the mismatched points are shown in Table 7.

When the number of pyramid layers is 8 , the relationship between the scale parameters of the pyramid image from 1.1, 1.2, .., 2.0 and the feature detection time is shown in Fig. 26.

When the number of pyramid layers is 8 , the relationship between the scale parameters of the pyramid images from 1.1, 1.2,..., 2.0 and the matching accuracy of the feature points is shown in Fig. 27.

When the number of pyramid layers is 8 , when the scale parameter of the pyramid image changes from 1.1 to 2.0, the correspondence between the number of feature point detection points and the exact matching points is shown in Fig. 28.

Experimental summary:

1. As can be seen from Fig. 26, the image of different scales (the difference between the left image and the right image scale is 50\%) and the pyramid layer is 8 . When the scale parameter between the pyramid images changes from 1.1 to 2.0, the time consumption of the algorithm gradually decreases. The maximum time is $219 \mathrm{~ms}$ and the minimum time is $78 \mathrm{~ms}$.

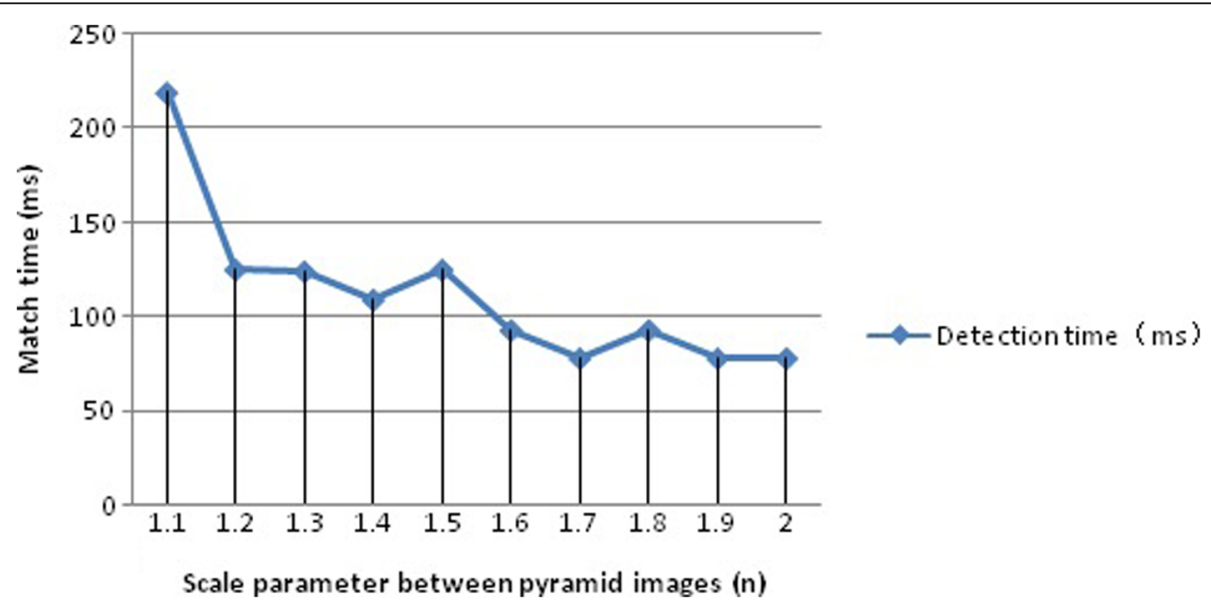

Fig. 26 Relationship between scale parameter variation and matching time between pyramid images 


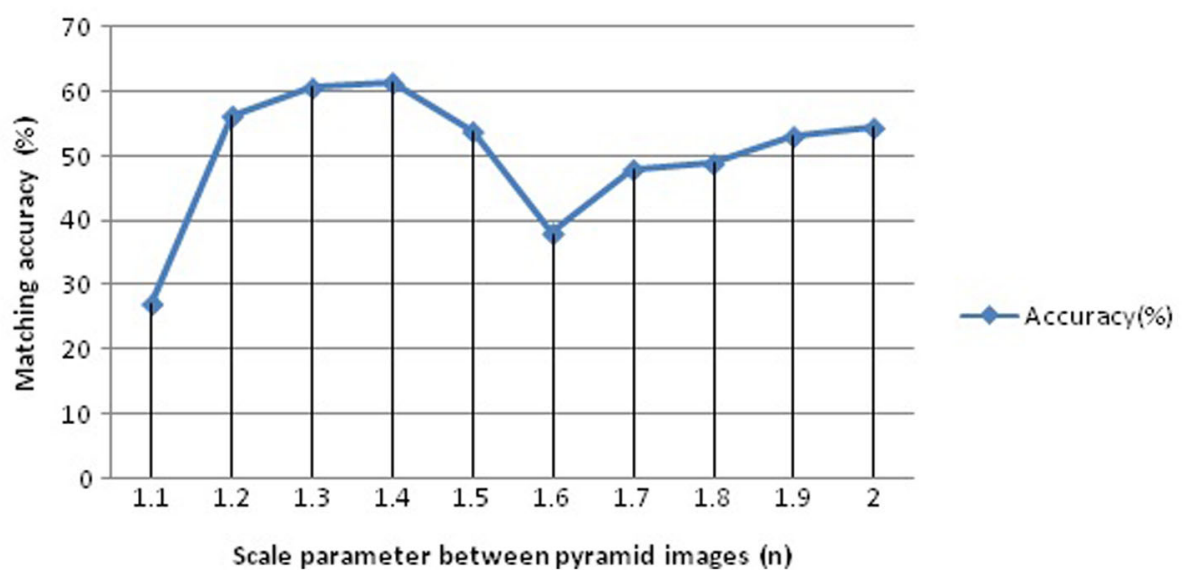

Fig. 27 Relationship between scale parameter variation and matching accuracy rate of pyramid image

2. As can be seen from Fig. 27, for the images of different scales (the difference between the left image and the right image scale are 50\%) and the pyramid layer is 8 . When the scale parameter between the pyramid images is 1.2-1.5 and 1.9, 2.0, the feature detection accuracy exceeds $50 \%$.

3. As can be seen from Fig. 28, for the images of different scales (the difference between the left image and the right image scale are 50\%) and the pyramid layer is 8 . When the scale parameter between the pyramid images changes from 1.1 to 2.0, the number of feature points and the number of exact matches are larger. The number of detected feature points is proportional to the number of accurately matching feature points.

(2) The second case: from the experimental results of the previous case, the accuracy rate is higher when the scale parameter of the pyramid image is 1.4, but this method is time consuming. Since this paper mainly studies the optimization method to improve the accuracy of feature detection, the feature detection experiment of different pyramid layer numbers $i(i=1,2, \ldots, 9)$ is selected by selecting the scale parameter between pyramid images as 1.4. Finally, the mismatch point is filtered out by the RANSAC algorithm with a threshold of 5 . The experimental results are shown in Table 8.

When the scale parameter between pyramid images is 1.4, the relationship between the number of pyramid layers and the detection time is shown in Fig. 29.

When the scale parameter between the pyramid images is 1.4 , the relationship between the continuous change of the pyramid layer and the detection accuracy is shown in Fig. 30.

When the scale parameter between the pyramid images is 1.4, the relationship between the number of feature point detection points and the exact matching

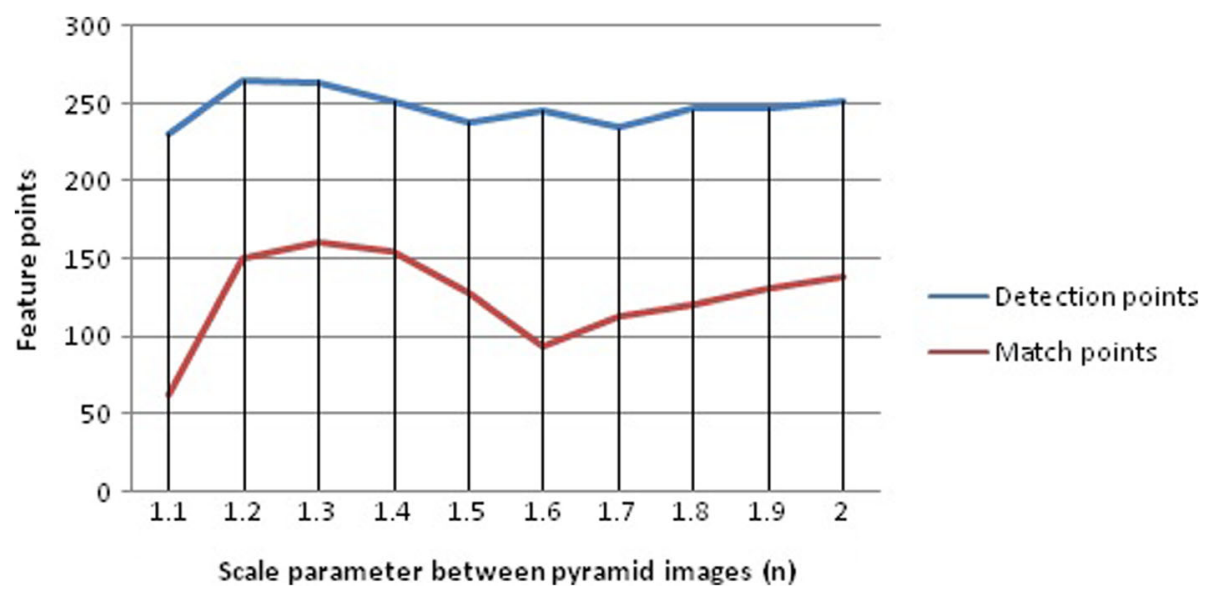

Fig. 28 Comparison of the number of feature points detected and the exact number of matches between the scale parameters of the pyramid image 
Table 8 Statistical results of experimental results of pyramid layer transformation when the scale parameter between pyramid images is 1.4

\begin{tabular}{lllll}
\hline Pyramid layer $(i)$ & Time $(\mathrm{ms})$ & Detection points & Match points & Accuracy $(\%)$ \\
\hline 1 & 63 & 282 & 10 & 3.6 \\
2 & 78 & 281 & 14 & 5.0 \\
3 & 109 & 289 & 96 & 33.2 \\
4 & 140 & 292 & 129 & 44.2 \\
5 & 141 & 285 & 150 & 52.6 \\
6 & 94 & 278 & 164 & 59.0 \\
7 & 78 & 262 & 159 & 60.7 \\
9 & 109 & 251 & 154 & 61.4 \\
\hline
\end{tabular}

points is shown in Fig. 31 during the change of the number of pyramid layers from 1 to 9 .

Experimental summary:

1. As can be seen from Fig. 29, for images with different scales (the difference between the left image and the right image scale is 50\%), the scale parameter between pyramid images is 1.4, and the algorithm takes a little time to increase the number of pyramid layers from 1 to 9 . When the number of pyramid layers is 4 or 5 , the algorithm takes a relatively long time.

2. As can be seen from Fig. 30, for images with different scales (the difference between the left image and the right image scale is $50 \%$ ), when the scale parameter between pyramid images is 1.4 , the accuracy of the matching with the number of pyramid layers is higher and higher and finally approaches $60 \%$.

3. As can be seen from Fig. 31, the image size of the pyramid image is 1.4 , and the number of feature points is slightly reduced when the number of pyramid layers increases. However, the number of exact matches has increased significantly.

From the statistics of the above two cases, the following conclusions can be drawn:

(3) The ORB algorithm extracts and matches the image of different scales (the difference between the left image and the right image is $50 \%$ ). When the scale parameter between the pyramid images is 1.6, the matching accuracy is generally low when the pyramid layer increases from 1 to 9 . But better than the BRISK algorithm, the average matching accuracy is close to $50 \%$. When the scale parameter between pyramid images is 1.4 , the number of pyramid layers changes from 1 to 9 . As the number of pyramid layers increases, the matching accuracy increases significantly, with a maximum of $60 \%$.

\subsection{ORB algorithm optimization experiment}

After the algorithm is optimized, the image matching experiments of the same scale and different scales are

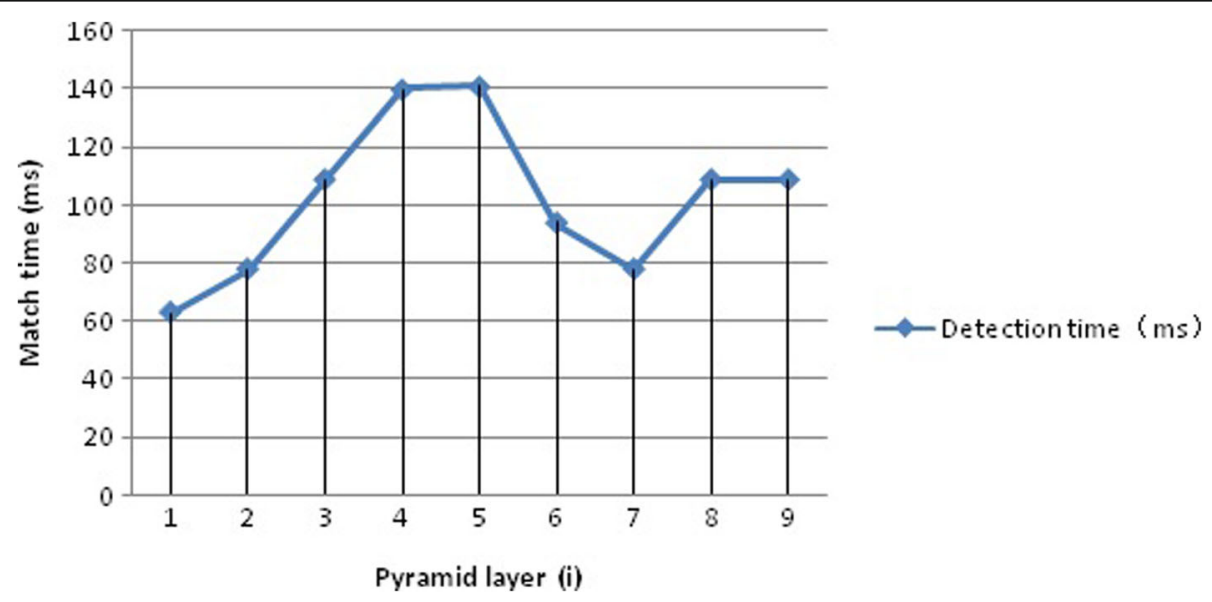

Fig. 29 Pyramid layer change and matching time relationship diagram 


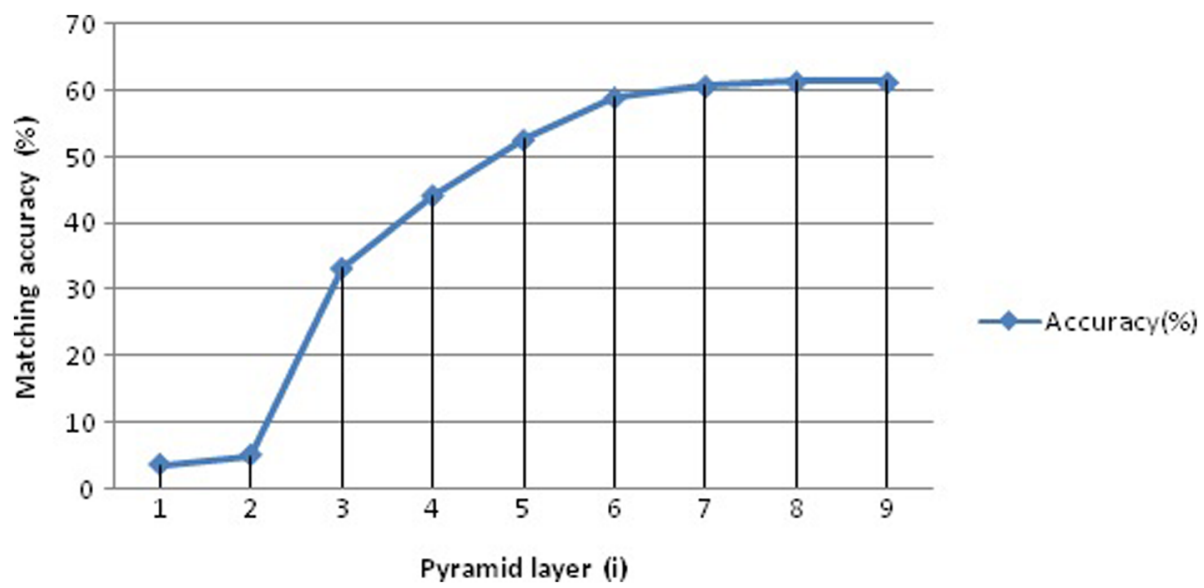

Fig. 30 Relationship between pyramid layer number change and matching accuracy

carried out, and compared with the previous experimental results.

(1) Image matching experiment at the same scale

When the scale parameter between the pyramid images is 1.1 and the number of pyramid layers is 8 , the Gaussian denoising process is performed after reading the image, and then the grayscale is performed. The experimental results after Gaussian filtering are shown in Fig. 32. The experimental results after grayscale are shown in Fig. 33.

The experimental results before and after the feature detection and filtering mismatch are shown in Fig. 34.

(2) Image matching experiment at different scales

The right image is reduced by $50 \%$ at different scales, and the algorithm is experimented by taking the scale parameter between the pyramid image as 1.4 and the number of pyramid layers as 8. And summarize the relevant experimental results.

(3) The experimental results before and after the algorithm optimization are shown in Table 9.

It can be seen from Table 9 that after Gaussian denoising and grayscale optimization, the number of feature points and the accuracy of feature points detected by the ORB algorithm are improved, indicating that the algorithm optimization has a certain effect. Of course, part of the time is lost as a cost. If you want to pursue fast processing, you do not need to perform denoising and graying. If you are pursuing precision, you can perform related optimization.

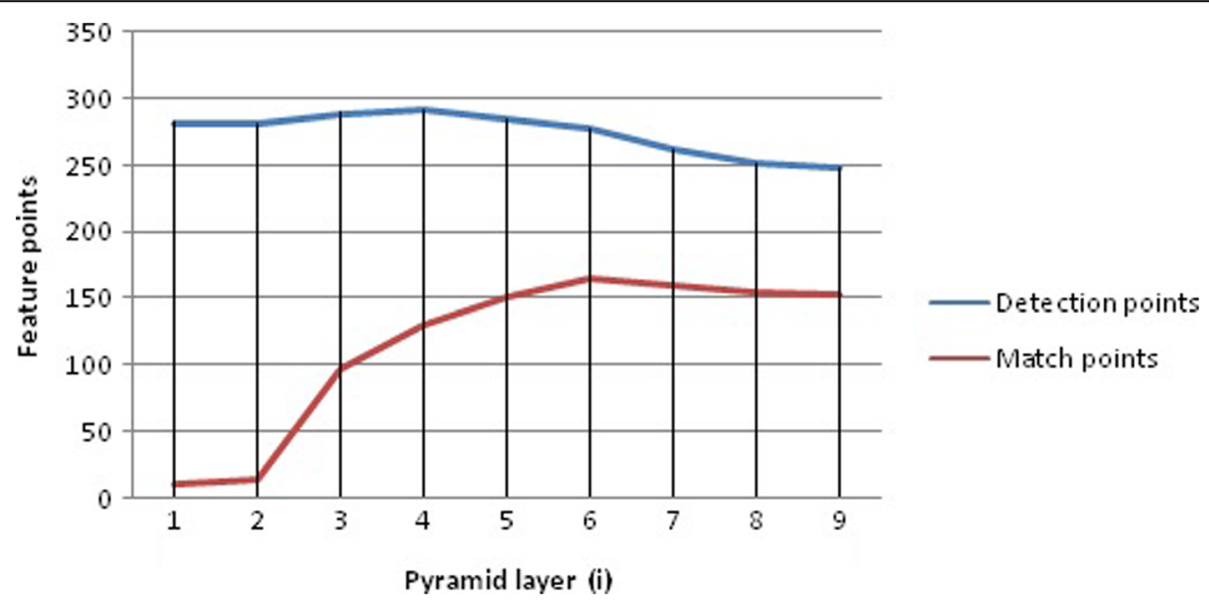

Fig. 31 Comparison of the number of feature points detected and the number of exact matches when the number of pyramid layers changes 

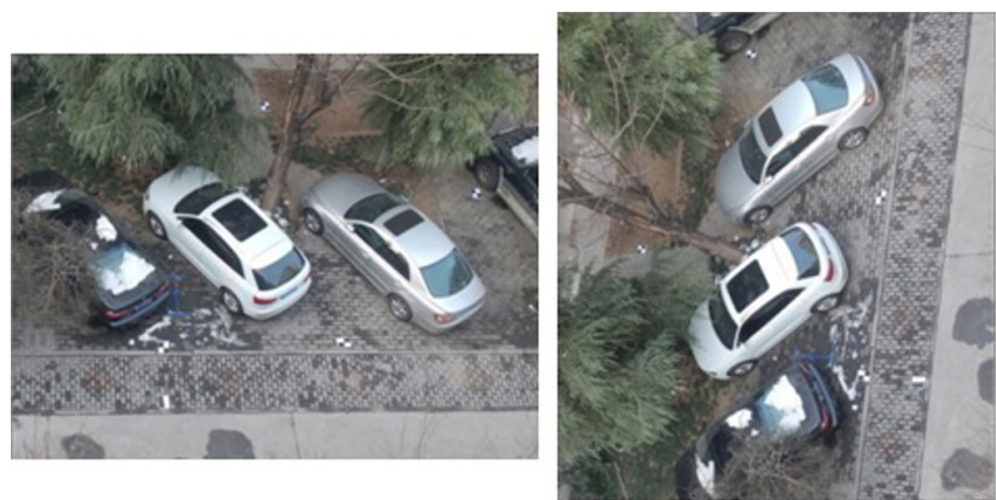

Fig. 32 Gaussian filtered image

\section{Discussion and experiments}

\subsection{Experimental analysis of feature points extraction on} the same scale

\section{(1) Experimental analysis of BRISK algorithm}

In the case of feature point matching on the same scale image, when the number of pyramid layers is unchanged $(i=4)$ and the scale parameters between pyramid images are gradually increasing, the following conclusions are drawn: first, the algorithm consumes a slight decrease in time consumption; and second, the accuracy of feature detection is gradually increasing and it tends to be stable from 37 to $54 \%$. The best matching accuracy is achieved when the scale parameter between pyramid images is 1.6. Third, the number of feature points detected is gradually decreasing, and the number of feature points that are accurately matched is gradually increasing.

When the scale parameter between pyramid images is $n=1.6$, there are several characteristics in the process of gradually increasing the number of pyramid layers: first, the consumption time of feature detection is gradually increasing. Second, the accuracy of feature detection is stable at around $55 \%$. Third, there is basically no change in the number of feature point detections and the number of feature points that are accurately matched.

\section{(2) Experimental analysis of ORB algorithm}

When the number of pyramid layers of the same scale image is 8 , the variation of the scale parameter $n$ between the pyramid images from $1.1,1.2, \ldots, 2.0$ has the following characteristics: first, the feature detection consumption time is gradually decreasing, which takes up to $234 \mathrm{~ms}$ and the minimum time is $94 \mathrm{~ms}$; and second, the accuracy of feature detection is gradually decreasing, from 96 to $87 \%$. During the change of the inter-scale image parameters of pyramid images from 1.4 to 2.0 , the matching accuracy fluctuates greatly and fluctuates around $87 \%$. Third, the number of feature points and the number of exact matches are gradually decreasing, and the trend is reduced.
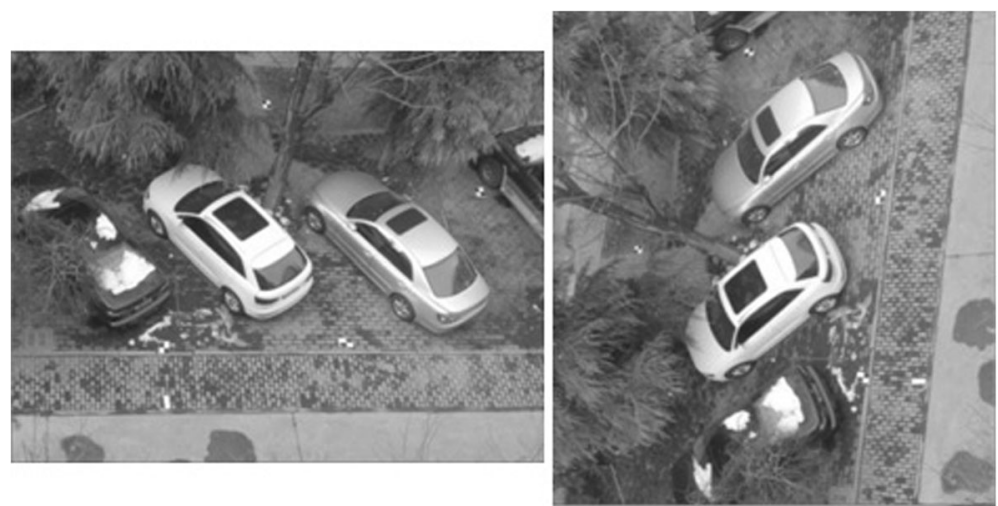

Fig. 33 Grayscale image 

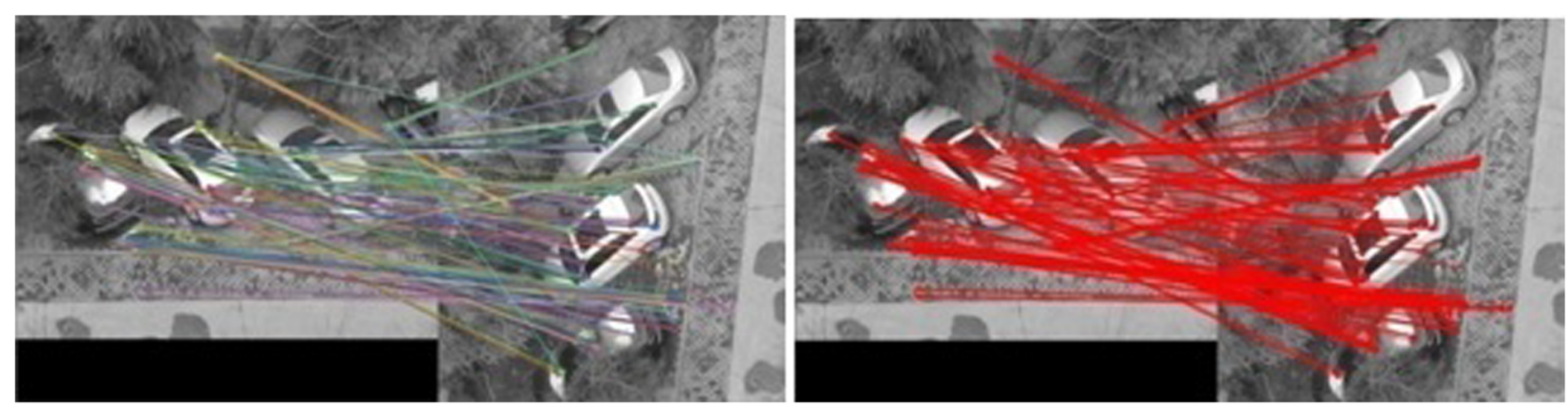

Fig. 34 Comparison of the effects before and after filtering the mismatch

When the scale parameter of the pyramid image of the same scale image is 1.1, the variation of the number of pyramid layers $i$ from 1 to 9 has the following characteristics: first, the feature detection consumption time is gradually increasing, the longest time is $281 \mathrm{~ms}$, and the minimum time is $109 \mathrm{~ms}$; second, the accuracy of feature detection is gradually increasing, with a minimum of $85 \%$ and a maximum of close to $97 \%$; and third, the number of feature points and the number of exact matches are relatively stable, basically forming a horizontal trend.

\subsection{Experimental analysis of feature points extraction from different scale images}

(1) Experimental analysis of BRISK algorithm

When the pyramid layer of the image is 4 at different scales (the difference between the left image and the right image scale is 50\%), the variation of the scale parameter between the pyramid images from 1.1 to 2.0 has the following characteristics: first, the scale parameter takes the least time between 1.5 and 1.8, and the maximum time is $343 \mathrm{~ms}$ and the minimum time is $156 \mathrm{~ms}$. Second, the accuracy of feature detection is very low. Except that the scale parameter between pyramid images is $16 \%$, the detection accuracy of other parameters is less than $10 \%$. Third, the number of feature points and the number of exact matches are very large. The algorithm has obvious defects, and there is much room for improvement.

When the scale parameter between the pyramid images is 1.6, the different scales (the left image and the right image scale differ by $50 \%$ ) have the following characteristics in the change of the image pyramid layer from 1 to 9: first, the feature point detection takes time and fluctuates greatly, with a maximum time of $312 \mathrm{~ms}$ and a minimum time of $172 \mathrm{~ms}$; second, when the pyramid layer is greater than or equal to 4 , the feature detection accuracy rate is always stable at about $16 \%$, and the feature detection accuracy of the pyramid layer is 1 to 3 layers is significantly increased with the increase of the number of layers; and third, the number of feature points and the exact number of matches are very large, and the algorithm has the defects of insufficient detection accuracy.

\section{(2) Experimental analysis of ORB algorithm}

The pyramid layer of different scale images (the difference between the left image and the right image scale is $50 \%$ ) is 8 , and the scale parameter between the pyramid images varies from 1.1 to 2.0. First, the time-consuming of the algorithm is gradually decreasing. The maximum time is $219 \mathrm{~ms}$, and the minimum time is $78 \mathrm{~ms}$. Second, when the scale parameter between pyramid images is

Table 9 Statistical results of experimental results before and after algorithm optimization

\begin{tabular}{|c|c|c|c|c|}
\hline Project & Feature points & Number of matches & Accuracy (\%) & Time (ms) \\
\hline \multicolumn{5}{|l|}{ Before optimization } \\
\hline Same scale & 348 & 336 & 96.6 & 234 \\
\hline Different scale & 251 & 154 & 61.4 & 109 \\
\hline \multicolumn{5}{|l|}{ After optimization } \\
\hline Gaussian denoising, grayscale & 363 & 353 & 97.3 & 296 \\
\hline Gaussian denoising & 354 & 346 & 97.7 & 265 \\
\hline Gaussian denoising, grayscale & 264 & 164 & 62.1 & 156 \\
\hline Gaussian denoising & 253 & 157 & 62.1 & 156 \\
\hline
\end{tabular}



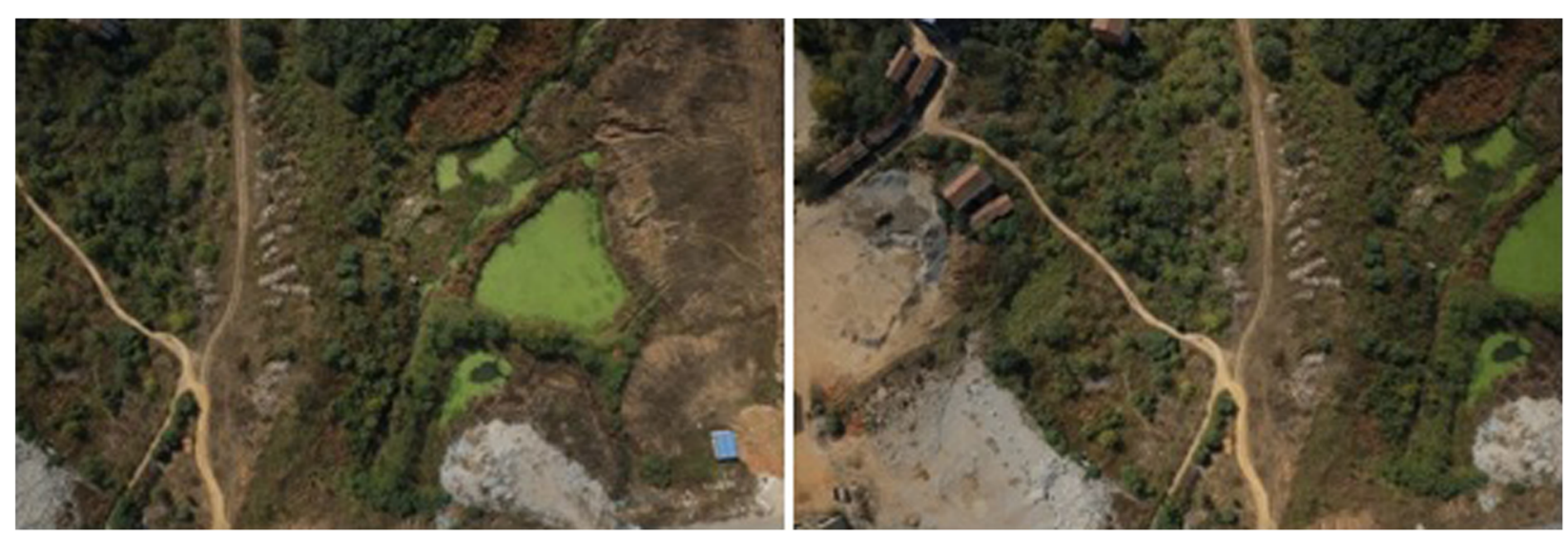

Fig. 35 Original image

$1.2-1.5$ and 1.9, 2.0, the accuracy of feature detection exceeds $50 \%$. Third, the number of feature points and the number of exact matches are large, and the number of detected feature points is proportional to the number of accurately matched feature points.

The scale parameter between the pyramid images is 1.4 , and the number of pyramid layers changes from 1 to 9 as follows: First, the overall time consumption of the algorithm increases slightly. When the number of pyramid layers is 4 or 5 , the algorithm takes a relatively long time. Second, as the number of pyramid layers increases, the matching accuracy becomes higher and higher, eventually approaching $60 \%$. Third, the number of feature points has a slight decrease, but the number of accurate matches has increased significantly.

\subsection{ORB algorithm optimization experiment analysis}

After the algorithm is optimized, the image matching experiment of the same scale and different scales is carried out. It can be seen from Table 9 that after the ORB algorithm performs Gaussian denoising and grayscale optimization, the number of detected feature points and the accuracy of feature points are improved, indicating that the algorithm optimization has a certain effect. Of course, part of the time is lost. If you want to pursue fast processing, you do not need to perform denoising and graying. If you are looking for precision, you can perform related optimization.

\section{Optimization algorithm application}

In order to verify the stitching effect of the ORB optimization algorithm, two adjacent drone images were selected for splicing experiments, and the experimental results were analyzed. The original dataset is shown in Fig. 35.

\subsection{Image stitching process}

Image stitching mainly includes image preprocessing, image registration, image synthesis, and other major links. The main steps of the splicing experiment are:

(1) Perform basic operation experiments such as enhancement transformation and smoothing on the

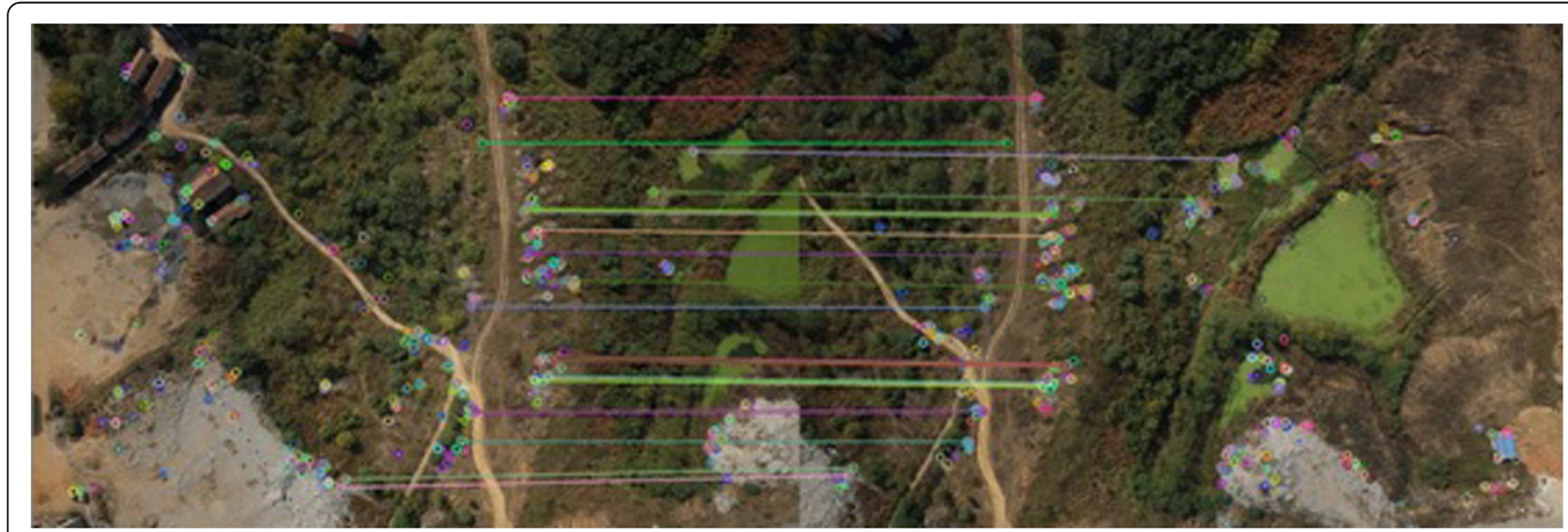

Fig. 36 Feature detection and feature point screening results 


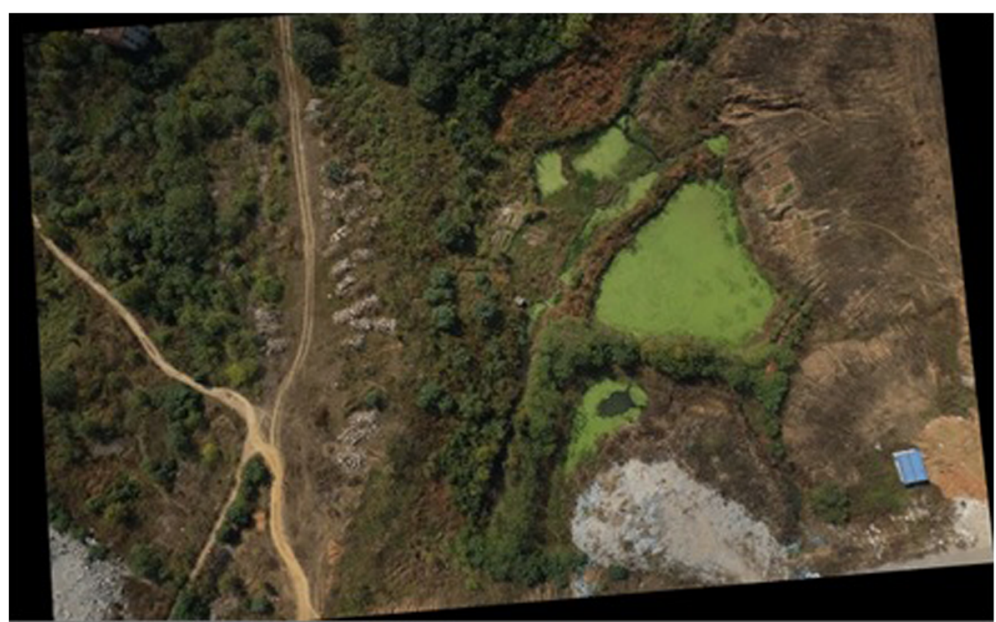

Fig. 37 Result graph of the right image after perspective transformation

original image to prepare for the next image mosaic.

(2) Image registration is the core of image stitching, and the accuracy of registration directly determines the stitching quality of images. Perform feature point detection and screen out accurate feature points. Accurately find the location of the feature points between the two images and establish an accurate conversion relationship model.

(3) After determining the conversion relationship model between the two images, the image to be stitched is mosaicked into a visually visible image according to the information of the overlapping area.

In the image feature point selection process, image Gaussian filtering is first performed and grayscaled. The feature point detection uses the RANSAC algorithm with a threshold of 5 to filter the mismatch points, ensuring the accuracy of the feature points used to establish the mathematical model.
The image fusion process uses a weighted smoothing algorithm to perform a fast and simple weighted smoothing algorithm to complete the stitching of the final image.

\subsection{ORB image mosaic implementation}

The experimental dataset is an aerial photography image of a certain area of the UAV. The left and right image aerial scales are basically the same, the image size is $612 * 408$, and the right image is slightly rotated.

(1) Firstly, the image to be stitched is read, and the RANSAC algorithm with a threshold of 5 is used to complete feature point matching and accurate matching point screening. The experimental results are shown in Fig. 36.

(2) Calculate the transformation matrix and complete the right image perspective matrix transformation. The transformation result is shown in Fig. 37.

(3) Create the stitched image range and complete the image stitching. The result is shown in Fig. 38.

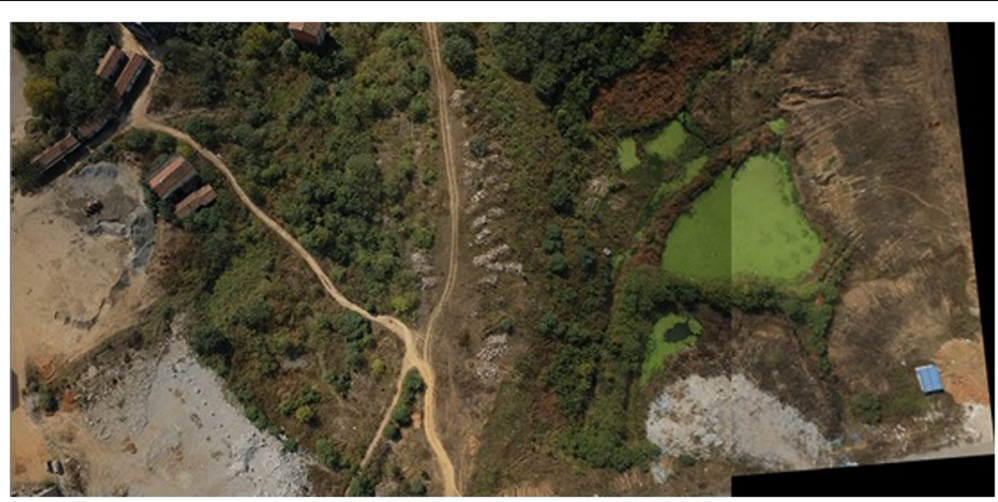

Fig. 38 Image mosaic effect 


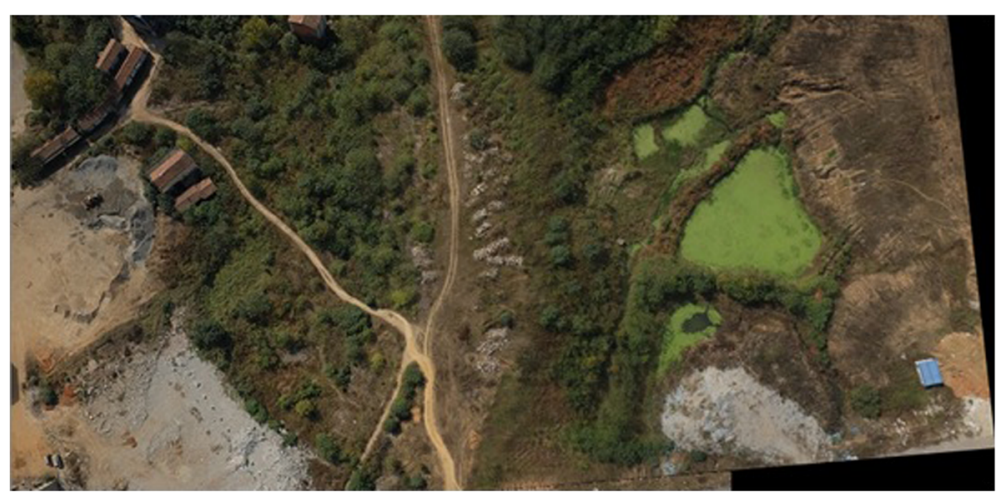

Fig. 39 Image after processing the stitching seam

(4) There is a clear stitching seam after the image is stitched, which is due to the color difference between the two images. Therefore, smoothing processing is required, and the processing of the stitching seam is quickly completed by the weighted smoothing algorithm. The result of the image stitching seam processing is shown in Fig. 39.

\section{Conclusions}

In this paper, the feature point matching based on BRISK and ORB algorithm and the algorithm improvement and feature point extraction experiment based on the combination of the two advantages are carried out. The main conclusions obtained through experiments are as follows:

(1) When the pyramid layer is 4 in the BRISK algorithm, the matching accuracy of the pyramid image between the data sources is high. In the case where the pyramid layer is 8 in the ORB algorithm, the matching accuracy of the pyramid image between the data sources is high. Therefore, the scale relationship between image sources has a direct impact on the accuracy of feature matching. It is recommended to prioritize the algorithm parameters before feature point extraction and matching.

(2) Combined with the characteristics of BRISK and ORB, the algorithm is optimized and improved, which makes the algorithm have both excellent illumination robustness and fast computing power, and BRISK scale invariance. Under the condition of optimal algorithm parameters, the number of feature points can be increased by $3 \%$, and the effective matching point can be improved by nearly $2 \%$.
(3) In this paper, the algorithm application experiment is carried out for the UAV image, and the feature extraction matching and image splicing work of the UAV image is completed. From the visual effect, the effect is good, and the optimization algorithm can be applied to the inorganic image processing application.

Abbreviations

3D: Three dimension; BRIEF: Binary robust independent elementary features; BRISK: Binary robust invariant scalable keypoints; FAST: Features from accelerated segment test; ORB: Object request broker; RANSAC: Random sample consensus; SIFT: Scale-invariant feature transform; SUSAN: Small univalue segment assimilating nucleus; UAV: Unmanned aerial vehicle

\section{Availability of data and materials}

We can provide the data.

Author's contribution

Wu did all the work. The author read and approved the final manuscript.

\section{Author's information}

Manyi Wu was born in 1983 and a Doctoral graduate student of Wuhan University, mainly concerned with UAV image processing and image feature extraction.

Ethics approval and consent to participate

Not applicable.

Consent for publication

Not applicable.

Competing interests

The author declare that he has no competing interests.

\section{Publisher's Note}

Springer Nature remains neutral with regard to jurisdictional claims in published maps and institutional affiliations.

Received: 29 July 2018 Accepted: 4 October 2018

Published online: 16 October 2018

\section{References}

1. F. Schaffalitzky, A. Zisserman, Multi-view Matching for Unordered Image Sets. In Proc. ECCV. (2002), pp. 414-431.

2. T. Tuytelaars, L. Van Gool, Matching widely separated views based on affine invariant regions. Int J Comput Vis 1(59), 61-85 (2004). 
3. K. Mikolajczyk, C. Schmid, Indexing Based on Scale Invariant Interest Points. Vol. 1. IEEE International Conference on Computer Vision, (2002), pp. 525-531.

4. C. Schmid, R. Mohr, Local Grayvalue invariants for image retrieval. IEEE Trans. Pattern Anal Machine Int 19(5), 530-534 (May 1997).

5. V. Ferrari, T. Tuytelaars, L. Van Gool, Simultaneous Object Recognition and Segmentation by Image Exploration, In Proc. Eighth European Conf. Computer Vision. (2004), pp. 40-54.

6. D. Lowe, Distinctive image features from scale-invariant Keypoints. Int'l J. Comp Vis 2(60), 91-110 (2004)

7. S. Lazebnik, C. Schmid, J. Ponce, Sparse Texture Representation Using AffineInvariant Neighborhoods. In Proc. Computer Vision and Pattern Recognition. (2003), pp. 319-324.

8. J. Sivic, A. Zisserman, Video Google: a Text Retrieval Approach to object Matching in Videos. In Proc. Ninth Int'l Conf. Computer Vision. (2003), pp. 1470-1478.

9. M. Brown, D. Lowe, Recognising Panoramas (Proc. Ninth Int'l Conf. Computer Vision, 2003), pp. 1218-1227.

10. R. Fergus, P. Perona, A. Zisserman, Object Class Recognition by Unsupervised Scale-Invariant Learning. In Proc. Conf. Computer Vision and Pattern Recognition. (2003), pp. 264-271.

11. D. Lowe, Distinctive image features from scale-invariant keypoints. Int'| Journal of Computer Vision 60(2), 91-110 (2004).

12. H. Bay, T. Tuytelaars, L.V. Gool, SURF: Speed Up Robust Features. Proc. of the European Conf. on Computer Vision. (2006), pp. 404-417.

13. H. Bay, A. Ess, T. Tuytelaars, L.V. Gool, Speeded-up robust features (SURF). Comput. Vis. Image Underst. 110(3), 346-359 (2008).

14. E. Rublee V. Rabaud, K. Konolige, et al, ORB: An Efficient Alternative to SIFT or SURF[C] (International Conference on Computer Vision, 2011), pp. 2564-2571.

15. S. Leutenegger, M. Chli, R.Y. Siegwart, BRISK: Binary Robust Invariant Scalable Keypoints [C]. International Conference on Computer Vision (IEEE, 2011), pp. 2548-2555.

16. E. ROSTEN, T. DRUMMOND, Fusing Points and Lines for High Performance Tracking $[C]$, vol 2 (Tenth IEEE International Conference on Computer Vision. IEEE, 2005), pp. 1508-1515.

17. S.U.N. Bo, Research on the Corner Detection Algorithm of Digital Images[D] (Hefei University of Technology, 2013)

18. L.I.A.N.G. Yan-ju, Q. Li, C.H.E.N. Da-peng, Y.A.N. Xue-jin, Fast and Robust LOG-FAST Corner Algorithm[J]. Computer Science 39(6), 251-254 (2012).

19. E. Rosten, R. Porter, T. Drummond, Faster and better: a machine learning approach to corner detection[J]. IEEE Trans. Pattern Anal. Mach. Intell. 32(1), 105-119 (2010).

20. E. Rosten, T. Drummond, Machine Learning for High Speed Corner Detection[C]//9th European Conference on Computer Vision (Springer, ACM, Berlin, Heidelberg, 2006), pp. 430-443.

21. M. Calonder, V. Lepetit, P. Fua, BRIEF: Binary Robust Independent Elementary Features (Proc. of the 11th European Conf. on Computer Vision, Heraklion, Crete, 2010), pp. 778-792. https://doi.org/10.1007/978-3-642-15561-1_56.

22. A. Buades, B. Coll, J.M. Morel, A Non-local Algorithm for Image Denoising[C]. vol 2. (IEEE Computer Society Conference, 2005), pp. 60-65.

23. A. Buades, B. Coll, J.M. Morel, A review of image denoising algorithms, with a new one[J]. Multiscale Model Simul 4(2), 490-530 (2005).

24. J. Weickert, B.M.T.H. Romeny, M.A. Viergever, Efficient and reliable schemes for nonlinear diffusion filtering[]]. Image Proc IEEE Trans 7(3), 398-410 (1998).

25. I. Pitas, A.N. Venetsanopoulos, Nonlinear Digital Filters[M] (Springer Science \& Business Media, 1990).

26. A. MARTIN, C.B. ROBERT, Random sample Concesus: proceeding of a parading for model fitting with applications to image analysis and automated cartography[J]. Commun. ACM 24(6), 381-395 (1981).

27. S. Qiang, Y. Yutang, S. Yuncen, et al., Fast and stable method based on optimized RANSAC algorithm for two-dimensional image[]]. China Comp. Federation Magazine 33(6), 2373-2377 (2012).

28. J. Jun, L. Zhijie, Y. Peng, ImprovedRANSACalgorithmofmatched points purifying[J]. J Xi'an Univ Architect Tech Nat Sci Edition 45(6), 896-901 (2013).

\section{Submit your manuscript to a SpringerOpen ${ }^{\circ}$ journal and benefit from:}

- Convenient online submission

- Rigorous peer review

- Open access: articles freely available online

- High visibility within the field

- Retaining the copyright to your article

Submit your next manuscript at $\boldsymbol{\nabla}$ springeropen.com 